Check for updates

Cite this: Phys. Chem. Chem. Phys., $2018,20,3184$

Received 6th September 2017. Accepted 12th October 2017

DOI: 10.1039/c7cp06068h

rsc.li/pccp

\section{Molecular details of the unique mechanism of chloride transport by a cyanobacterial rhodopsin $\dagger$}

\author{
Andrew Harris, (D a Mattia Saita, (D) ${ }^{b}$ Tom Resler, ${ }^{b}$ Alexandra Hughes-Visentin, (iD a \\ Raiza Maia, ${ }^{b}$ Franziska Pranga-Sellnau, (D) b Ana-Nicoleta Bondar, (D) c \\ Joachim Heberle (iD $*^{b}$ and Leonid S. Brown (ID *a
}

\begin{abstract}
Microbial rhodopsins are well known as versatile and ubiquitous light-driven ion transporters and photosensors. While the proton transport mechanism has been studied in great detail, much less is known about various modes of anion transport. Until recently, only two main groups of light-driven anion pumps were known, archaeal halorhodopsins (HRs) and bacterial chloride pumps (known as CIRs or NTQs). Last year, another group of cyanobacterial anion pumps with a very distinct primary structure was reported. Here, we studied the chloride-transporting photocycle of a representative of this new group, Mastigocladopsis repens rhodopsin (MastR), using time-resolved spectroscopy in the infrared and visible ranges and site-directed mutagenesis. We found that, in accordance with its unique amino acid sequence containing many polar residues in the transmembrane region of the protein, its photocycle features a number of unusual molecular events not known for other anion-pumping rhodopsins. It appears that light-driven chloride ion transfers by MastR are coupled with translocation of protons and water molecules as well as perturbation of several polar sidechains. Of particular interest is transient deprotonation of Asp-85, homologous to the cytoplasmic proton donor of light-driven proton pumps (such as Asp-96 of bacteriorhodopsin), which may serve as a regulatory mechanism.
\end{abstract}

\section{Introduction}

Microbial rhodopsins are a diverse family of retinal-binding membrane proteins that are expressed in organisms from all three kingdoms of life and can be found in aquatic and terrestrial ecosystems all over the world. They share a common 7TM alpha-helical structure with the retinal chromophore bound to the Schiff base lysine in the middle of the seventh helix. Microbial rhodopsins can function as light-driven ion transporters, including proton, chloride, and sodium pumps, as well as photosensors, light-gated cation and anion channels and light-activatable enzymes. ${ }^{3-5}$ Of these functions, light-driven proton transport has been studied the most thoroughly, mainly due to several decades of intensive research on bacteriorhodopsin (BR),$^{6-9}$ the first discovered haloarchaeal proton pump, supplemented by more recent studies of its fungal, algal, and

\footnotetext{
${ }^{a}$ Department of Physics and Biophysics Interdepartmental Group, University of Guelph, 50 Stone Road East, Guelph, Ontario N1G 2W1, Canada. E-mail: lebrown@uoguelph.ca

${ }^{b}$ Experimental Molecular Biophysics Group, Department of Physics, Freie Universität Berlin, Arnimallee 14, 14195 Berlin, Germany. E-mail:jheberle@zedat.fu-berlin.de

${ }^{c}$ Theoretical Molecular Biophysics Group, Department of Physics, Freie Universität Berlin, Arnimallee 14, 14195 Berlin, Germany

$\dagger$ Electronic supplementary information (ESI) available. See DOI: 10.1039/c7cp06068h
}

bacterial homologs. ${ }^{10-14}$ To better navigate the expanded spectrum of known microbial rhodopsin functionalities, it has recently become popular to label groups of microbial rhodopsins based on a three residue motif from the third TM helix (helix C). These three residues are homologous to the proton acceptor, the acceptor hydrogen-bonding partner, and the cytoplasmic proton donor from proton pumping rhodopsins (e.g., D85, T89, and D96 of BR make the DTD motif). ${ }^{12,15-17}$ According to this classification, most archaeal and fungal proton pumps have DTD motifs, while bacterial proton pumps (proteorhodopsins and xanthorhodopsins) most commonly display DTE, even though some cases of DTK and DTG proton pumps have been identified. ${ }^{18,19}$ In contrast, lightdriven sodium pumps have NDQ motifs, in which the primary proton acceptor of the Schiff base shifts to the second position. ${ }^{20}$

Compared to light-driven cation transport, anion transport by microbial rhodopsins has not been studied as extensively. Originally, inward light-driven chloride ion transport was discovered in Halobacteria and was found to be performed by halorhodopsin (HR). ${ }^{22}$ Halorhodopsins are TSA motif rhodopsins closely related to $\mathrm{BR}$, even though their exact evolutionary relationship is not clear. ${ }^{23-25} \mathrm{X}$-ray structures and mutagenesis studies of HR from Natronobacterium pharaonis (pHR) and Halobacterium salinarium ( $\mathrm{SHR}$ ) showed that a chloride ion is bound in the vicinity of the retinal Schiff base and is coordinated by multiple hydrogen bonds, including those with polar sidechains of 
the first two members of the TSA motif. ${ }^{26,27}$ Even though replacement of the motif-constituting threonine and serine (T126 and S130 of pHR, T111 and S115 of sHR) affects chloride binding, none of them seem to be indispensable for chloride transport, with the serine being more important than the threonine. ${ }^{28,29}$ Interestingly, introduction of threonine into the first position of the DTD motif of BR converts it into a chloride pump, ${ }^{30,31}$ while the reverse conversion of $\mathrm{pHR}$ into a proton pump could not be achieved. ${ }^{32,33}$ The X-ray structures of several photocycle intermediates for pHR and sHR give some insights into the chloride translocation mechanism. ${ }^{34,35}$ They identify a transient chloride binding site in the $\mathrm{N}$ intermediate of $\mathrm{pHR}$, in which chloride is translocated to the cytoplasmic side of the Schiff base, but stays in its vicinity, coordinated by the motifforming serine (S130 of pHR) and water molecules. It appears that a chloride ion is released to the cytoplasmic side from this site upon the decay of the $\mathrm{N}$ (also known as L2) intermediate, ${ }^{36,37}$ the process in which another threonine residue (T203 in SHR, T218 in pHR) plays an important role. ${ }^{28,38}$ It should be mentioned that chloride ion release may be coupled to the movement of a proton, but the exact source of this proton is not known. ${ }^{35,39}$ Chloride uptake from the extracellular side occurs upon the decay of the $\mathrm{O}$ intermediate. ${ }^{35-37}$

The second distinct group of inward chloride transporting rhodopsins (so-called ClRs) have been identified fairly recently, originally in Flavobacteria. ${ }^{16,40}$ These rhodopsins have low sequence homology to HRs, possess an NTQ motif, and are closely related to the NDQ sodium pumps and DTE proton-pumping xanthorhodopsins. ${ }^{25}$ Two X-ray structures of a representative of this group show that, similar to HRs, a chloride ion is coordinated by the first two members of the motif, Asn and Thr, and that the latter is more critical for chloride binding. ${ }^{41,42}$ The detailed mechanism of chloride transport is not known, and the HR-like sequence of chloride uptake and release was suggested for some members of this group but not for the others. ${ }^{40,43}$

Finally, the most recent addition to the ranks of anion transporting microbial rhodopsins is a distinct group of cyanobacterial light-driven anion pumps with the TSD motif. ${ }^{44,45}$ It should be noted that the motif-forming Asp is not strictly conserved and can be present as Val, Leu, or Ile in some members of the group (Fig. S1, ESI $\dagger$ ). Phylogenetic analysis and sequence comparison (Fig. 1 and Fig. S1, ESI $\dagger$ ) suggest that this group is distinct both from TSA HRs and NTQ ClRs, even though it clearly belongs to the archaeal-like half of the phylogenetic tree. The first characterized member of the TSD/ V/L/I group is from Mastigocladopsis repens (named MrHR). ${ }^{44}$ Interestingly, in contrast to HR, this chloride pump could be converted to a proton pump by a single mutation of the first member of the TSD motif into Asp. The second characterized member of the group from Synechocystis sp. PCC 7509 (SyHR) showed broader anion specificity, being able to transport not only halides but sulfate ions as well. ${ }^{45}$ Here, we will report on unique aspects of the photocycle and the mechanism of ion transport by the former, cyanobacterial anion pump from Mastigocladopsis repens, which we prefer to call MastR rather than MrHR, to stress its differences from the haloarchaeal

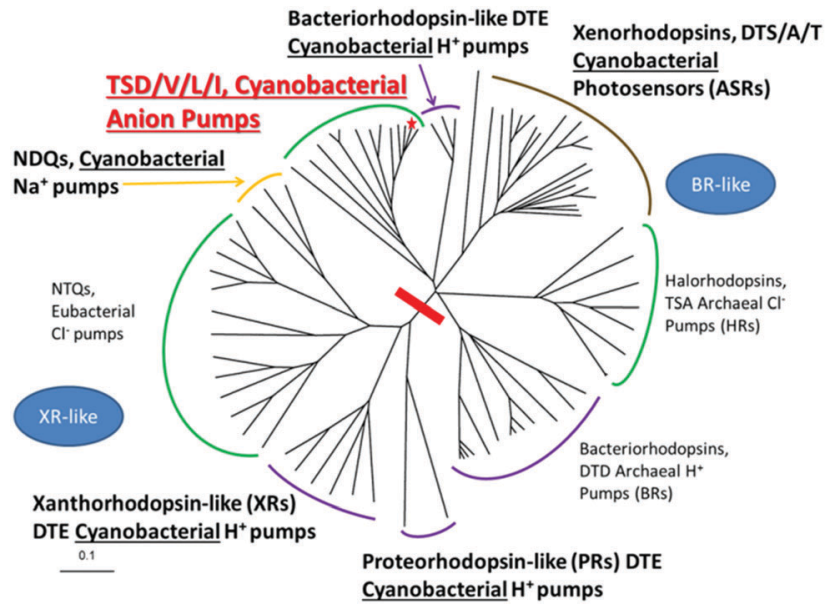

Fig. 1 Relationship of the new cyanobacterial group of anion transporters (labeled red) to other cyanobacterial, haloarchaeal and chloride-transporting rhodopsins. CLUSTAL Omega ${ }^{2}$ phylogenetic tree of selected functional groups of microbial rhodopsins with the helix $\mathrm{C}$ motifs indicated, rendered by TreeView. ${ }^{21}$ MastR is shown by the red asterisk. The red bar in the center of the tree separates archaeal-like rhodopsins (BR-like) and bacterial-like (proteorhodopsin- and xanthorhodopsin-like) clusters. Cyanobacterial rhodopsins are labeled in bold, anion pumps have green arcs, proton pumps - purple arcs, sodium pumps - yellow arc, and photosensors - tan arc.

chloride pumps. Using time-resolved spectroscopy in the infrared and visible ranges and site-directed mutagenesis we found that light-driven chloride ion transfers by MastR are coupled to translocation of protons and water molecules as well as perturbation of several polar sidechains. Perhaps most interestingly, we detected transient deprotonation of the third member of the TSD motif (Asp-85) which may serve as a regulatory mechanism.

\section{Experimental}

\subsection{Protein expression}

Genes encoding the wild-type (Genbank accession: WP_017314391) and mutant MastR were cloned into pET21a(+) vector (EMD Millipore, Billerica, MA) by GenScript (Piscataway, NJ) using NdeI-XhoI restriction sites, which added C-terminal $6 \times$ His-tag after the LE insert. Escherichia coli C41(DE3) OverExpress Chemically Competent Cells (Lucigen, Middleton, WI) were transformed with the plasmids using Lucigen's heat-shock transformation protocol. After thawing the E. coli C41 cells on ice for 10-15 min, $50 \mathrm{ng}$ of DNA was added to $50 \mu \mathrm{L}$ of cells and incubated on ice for $30 \mathrm{~min}$. The cells were heat shocked in a $42{ }^{\circ} \mathrm{C}$ water bath for $45 \mathrm{~s}$, and then incubated on ice for $2 \mathrm{~min}$. The cells were added to $950 \mu \mathrm{L}$ of $2 \times$ YT media ( $1 \%$ yeast extract, $1.6 \%$ tryptone, $1 \% \mathrm{NaCl}$ ) and incubated at $37{ }^{\circ} \mathrm{C}$ and $250 \mathrm{rpm}$ shaking for $1 \mathrm{~h} .100 \mu \mathrm{L}$ of cell culture was spread onto YT media agar plates (YT media with $1.5 \%$ agar and $0.1 \mathrm{mg} \mathrm{mL}$ ampicillin at pH 7.0) and incubated overnight at $37{ }^{\circ} \mathrm{C}$. During cell growth stages, $0.1 \mathrm{mg} \mathrm{mL^{-1 }}$ ampicillin was always added to the $2 \times$ YT media. For the small scale colony screening, six isolated bacterial colonies were selected to inoculate $2 \mathrm{~mL}$ of $2 \times$ YT media for incubation at $37{ }^{\circ} \mathrm{C}$ and $240 \mathrm{rpm}$ overnight and 
transferred to $25 \mathrm{~mL} 2 \times$ YT media. When the $25 \mathrm{~mL}$ culture reached an optical density at $600 \mathrm{~nm}$ (OD600, measured by Cary50, Varian) of $\sim 0.4$, it was induced by $1 \mathrm{mM}$ isopropyl- $\beta$-Dthiogalactoside (IPTG) and $7.5 \mu \mathrm{M}$ all-trans retinal. The induced cell culture was incubated for $3 \mathrm{~h}$ at $37{ }^{\circ} \mathrm{C}$ and $240 \mathrm{rpm}$. The cells were collected through low-speed centrifugation at $4680 \times g$ and $4{ }^{\circ} \mathrm{C}$. The intensity of colour between the cell pellets was compared, and the colony with the most intense pink colour and largest pellet was selected as the best protein expressing colony. The best protein expressing colony was grown in $1 \mathrm{~L}$ of $2 \times$ YT media using shake flask cultures. The colony material was used to inoculate $2 \mathrm{~mL}$ of media and was incubated overnight at $37{ }^{\circ} \mathrm{C}$ and $240 \mathrm{rpm}$. The $2 \mathrm{~mL}$ cell culture was used to inoculate $25 \mathrm{~mL}$ of media which was then incubated overnight at $37{ }^{\circ} \mathrm{C}$ and $240 \mathrm{rpm}$. The $25 \mathrm{~mL}$ cell culture was used to inoculate $1 \mathrm{~L}$ of media and was incubated at $37^{\circ} \mathrm{C}$ and $240 \mathrm{rpm}$. Once an OD600 of $\sim 0.4$ was reached, the $1 \mathrm{~L}$ cell culture was induced with a total concentration of $1 \mathrm{mM}$ IPTG and $7.5 \mu \mathrm{M}$ all-trans retinal and was incubated for $3 \mathrm{~h}$ at $37{ }^{\circ} \mathrm{C}$ and $275 \mathrm{rpm}$. The cells were collected with low-speed centrifugation at $4680 \times g$ and $4{ }^{\circ} \mathrm{C}$ for $10 \mathrm{~min}$. The cell pellet was resuspended in $150 \mathrm{mM} \mathrm{NaCl}$ and was centrifuged again at $4680 \times g$ and $4{ }^{\circ} \mathrm{C}$ for $10 \mathrm{~min}$. The cell pellet was re-suspended in $40 \mathrm{~mL}$ of lysis buffer $\left(150 \mathrm{mM} \mathrm{NaCl}, 0.05 \mathrm{M}\right.$ Tris base, $1 \mathrm{mM} \mathrm{MgCl}_{2}, 2 \mu \mathrm{g} \mathrm{\textrm {mL } ^ { - 1 }}$ DNase I, $0.2 \mathrm{mg} \mathrm{mL}^{-1}$ lysozyme, $\mathrm{pH}=7.2$ ). The suspended cells were left to shake (IKA VIBRAX) at $400 \mathrm{rpm}$ at room temperature for $3 \mathrm{~h}$ and frozen for future use. The functionality of $E$. coli expressed MastR was confirmed by the whole cell ion transport assays (see Fig. S2 and Supplementary methods, ESI $\dagger$ ).

\subsection{Sample preparation and experimental set-up for time-resolved spectroscopy in the visible range}

The thawed cells were subjected to sonication (Fisher Sonic Dismembrator Model 500) to produce fragments of inner membranes suitable for spectroscopy in the visible range. The sonication regime was optimized to produce fragments of membranes small enough to minimize light scattering, which required longer and stronger sonication compared to the one used previously. ${ }^{18}$ We compared the photocycles of MastR in the membranes obtained using the modified sonication protocol with the earlier one to make sure the protein was not damaged as a result of more intense sonication. The sonicated sample was spun down at $4680 \times g$ and $4{ }^{\circ} \mathrm{C}$ for $10 \mathrm{~min}$ to get rid of unbroken cells and the supernatant was collected. The membrane fragments were then sedimented with the ultracentrifuge at $150000 \times g$ and $4{ }^{\circ} \mathrm{C}$ for $1 \mathrm{~h}$, and a soft upper part of the pellet containing smaller membrane fragments was collected. This membrane fraction suspension was then centrifuged at $32600 \times g$ for $10 \mathrm{~min}$ to remove larger membrane fragments.

Flash photolysis experiments were run on the membranes encased within polyacrylamide gels (Fig. S3, ESI $\dagger$ ). ${ }^{46}$ The gels were prepared using $700 \mu \mathrm{L}$ of the membrane suspension, $300 \mu \mathrm{L}$ of $33 \%$ acrylamide and $1 \%$ bisacrylamide solutions mixture, $2.4 \mu \mathrm{L}$ of $10 \%$ ammonium persulfate, and $3 \mu \mathrm{L}$ of $N, N, N^{\prime}, N^{\prime}$-tetramethylethylenediamine. After solidification, the gels were washed with $100 \mathrm{mM} \mathrm{NaCl}$ for at least $4 \mathrm{~h}$ at room temperature, and stored at $4{ }^{\circ} \mathrm{C}$ in $\mathrm{pH} 6$ buffer (the following buffer was used unless indicated otherwise: $50 \mathrm{mM} \mathrm{KH}_{2} \mathrm{PO}_{4}$, $50 \mathrm{mM}$ MES, $100 \mathrm{mM} \mathrm{NaCl}, \mathrm{pH}$ 6). If different $\mathrm{pH}$ and/or salt conditions were required, the storage buffer was replaced by soaking the gel in an appropriate buffer $(100 \mathrm{~mL}$ or more) for at least 90 minutes prior to the measurement.

Flash-photolysis spectroscopy was performed using a custombuilt single-wavelength spectrometer described elsewhere. ${ }^{47}$ In brief, the photocycle was initiated with 7 ns pulses of the second harmonic of an Nd-YAG laser at $532 \mathrm{~nm}$ (Continuum Minilite II). Absorption changes of the monochromatic light (provided by an Oriel QTH source and two monochromators) were followed using an Oriel photomultiplier, an amplifier with a $350 \mathrm{MHz}$ bandwidth, and a Gage AD converter (CompuScope 12100-64M). Kinetic traces were averaged (normally, 200-1000 traces) and converted into a quasi-logarithmic time scale using in-house software. Global multi-exponential analysis was performed by FITEXP. ${ }^{48}$

For the FTIR samples, the flash-photolysis spectroscopy in the visible range was performed using a commercial setup (LKS80, Applied Photophysics) as described earlier. ${ }^{49}$ Briefly, the sample was excited with $10 \mathrm{~ns}$ pulses at $532 \mathrm{~nm}$ by the emission of an OPO (optical parametric oscillator) driven by the third harmonics of an Nd-YAG laser (Quanta Ray Lab 150, Spectra physics). The energy density at the sample was adjusted to $3 \mathrm{~mJ} \mathrm{~cm}{ }^{-2}$.

\subsection{Sample preparation and experimental set-up for Raman and FTIR spectroscopy}

Vibrational spectroscopy was performed on His-tag-affinity purified lipid-reconstituted samples. The membrane pellet was re-suspended in solubilization buffer $(50 \mathrm{mM} \mathrm{NaCl}$, $5 \mathrm{mM}$ Tris base, 1\% DDM, pH = 7.5) and was left to stir overnight at $4{ }^{\circ} \mathrm{C}$ in the dark. The insoluble membrane debris was removed with the ultracentrifuge at $150000 \times g$ and $4{ }^{\circ} \mathrm{C}$ for $1 \mathrm{~h}$. The supernatant was collected, and the protein yield was estimated spectrophotometrically (Cary50) using the extinction coefficient of ASR, ${ }^{50}$ to determine the amount of nickel-nitrilotriacetic acid $\left(\mathrm{Ni}^{2+}-\mathrm{NTA}\right)$ resin (Qiagen) to be added. The resin was washed with $\sim 200 \mathrm{~mL}$ of $150 \mathrm{mM} \mathrm{NaCl}$ on a membrane filter (Thermo Scientific Nalgene MF75 filter, $0.8 \mu \mathrm{m}$ pore size), re-suspended in $10 \times$ binding buffer $(3 \mathrm{M} \mathrm{NaCl}, 0.5 \mathrm{M}$ Tris base, $0.05 \%$ DDM, $\mathrm{pH}=8$ ), and added to the solubilized protein sample to stir overnight in the $4{ }^{\circ} \mathrm{C}$ cold room. The membrane filter was used to wash the protein bound to the resin approximately eight times with $25 \mathrm{~mL}$ of washing buffer $(0.3 \mathrm{M} \mathrm{NaCl}$, $0.05 \mathrm{M}$ Tris, $40 \mathrm{mM}$ imidazole, $0.05 \% \mathrm{DDM}, \mathrm{pH}=8$ ) to remove any contaminating proteins. The washed protein-resin sample was collected by low-speed centrifugation at $1404 \times g$ and $4{ }^{\circ} \mathrm{C}$ for $5 \mathrm{~min}$. The supernatant was discarded and $5-10 \mathrm{~mL}$ of elution buffer (0.3 M NaCl, 0.05 M Tris, 0.5 M imidazole, 0.05\% DDM, $\mathrm{pH}=8$ ) was added and stirred on ice for $10 \mathrm{~min}$. Typically a total of $50 \mathrm{~mL}$ of eluted protein in elution buffer was collected, and this sample was syringe filtered $(0.22 \mu \mathrm{m}$ pore size $)$ to remove any large debris or accidentally collected resin. An Amicon Ultra $15 \mathrm{~mL}$ centrifugal filter tube was used to concentrate the sample 
down to a small volume, through repeated centrifugation at $4000 \times g$ and $4{ }^{\circ} \mathrm{C}$ for $20 \mathrm{~min}$. The buffer was exchanged to reconstitution buffer ( $50 \mathrm{mM} \mathrm{NaCl}, 10 \mathrm{mM}$ Tris, 0.05\% DDM, $\mathrm{pH}=8$ ) through washing it with a total volume of $50 \mathrm{~mL}$ by repeated centrifugation in the filter tube. For Raman and FTIR spectroscopy, the liposomes used for reconstitution were made of 1,2-dimyristoyl-sn-glycero-3-phosphocholine (DMPC) and 1,2-dimyristoyl-sn-glycero-3-phosphate (DMPA) in a 9:1 w/w ratio. The lipids were dissolved in chloroform, stirred for $90 \mathrm{~min}$, and the chloroform was removed under vacuum inside a desiccator for at least $4 \mathrm{~h}$. The lipid film was rehydrated with $1 \mathrm{~mL}$ of reconstitution buffer to form an $11.1 \mathrm{mg} \mathrm{mL}^{-1}$ suspension. The solubilized, concentrated pure protein was combined with the lipid suspension at a $0.73: 1$ protein to lipid ratio, and Triton $\mathrm{X}$-100 stock ( $\left.0.2 \mathrm{mg} \mu \mathrm{L}^{-1}\right)$ was added to the sample to the final concentration of $0.8 \mathrm{mg} \mathrm{mL}^{-1}$, at which a noticeable decrease in turbidity was observed. After $6 \mathrm{~h}$ of stirring at $4{ }^{\circ} \mathrm{C}, 0.6 \mathrm{~g} \mathrm{~mL}^{-1}$ of BioBeads SM-2 (Bio-Rad) was added to the sample to absorb the detergent, and the sample was mixed on the Orbitron Rotator for 24 h. A 27 G needle (BD) was used to remove the reconstituted protein and the BioBeads were washed with reconstitution buffer to collect all reconstituted protein. The sample was centrifuged at $150000 \times g$ and $4{ }^{\circ} \mathrm{C}$ for $1 \mathrm{~h}$ to collect proteoliposomes.

Raman spectra were collected using $5 \mu \mathrm{L}$ of a wet paste of the proteoliposomes hydrated with desired buffer using FRA106/s accessory to the Bruker IFS66vs spectrometer, with Nd-YAG laser excitation at $1064 \mathrm{~nm}$, at a $2 \mathrm{~cm}^{-1}$ resolution, with the OPUS software.

FTIR transmission difference spectroscopy was performed at $22{ }^{\circ} \mathrm{C}$ using Bruker Vertex80v spectrometer, ${ }^{51}$ in three different regimes (steady-state, rapid scan, and step-scan). Broadband time-resolved FTIR experiments with $\mu$ s time resolution were performed by applying the step-scan technique as described previously. ${ }^{52}$ The step-scan data have been treated with SVD analysis and reconstructed with the first 5 components. For ns time resolution, a home-built IR spectrometer based on tunable quantum cascade lasers (QCL) was employed. ${ }^{53} 5 \mu \mathrm{L}$ of MastR suspension (in $3 \mathrm{mM} \mathrm{KCl}$ and $3 \mathrm{mM}$ MES, pH 6) was dried on a $\mathrm{BaF}_{2}$ window to produce absorption of the amide $\mathrm{I}$ band of $\sim 1$ and rehydrated by 30 min equilibration with $4 \mu \mathrm{L}$ drop of $20 \%$ glycerol. The final buffer and salt concentrations are approximate due to the film drying effects (the $\mathrm{Cl}$ concentration is approximately $3 \mathrm{M}$ ). The replacement of $\mathrm{NaCl}$ by $\mathrm{KCl}$ was verified not to have any effects on the kinetics in E. coli membranes (not shown).

\section{Results and discussion}

\subsection{Characterization of the wild-type MastR photocycle by time-resolved spectroscopy in the visible range}

Previously, the photocycle of MastR (called MrHR) was characterized in a purified detergent-solubilized form in the presence of $100 \mathrm{mM} \mathrm{NaCl} .^{44}$ Similar to $\mathrm{pHR}$, the intermediates resembling $\mathrm{L} / \mathrm{N}$ (measured at $460 \mathrm{~nm}$ ), $\mathrm{O}$ (at $620 \mathrm{~nm}$ ), and a ground state-like $\mathrm{MrHR}^{\prime}$ (at $540 \mathrm{~nm}$ ) were detected, but no M-like intermediate with deprotonated Schiff base was found.

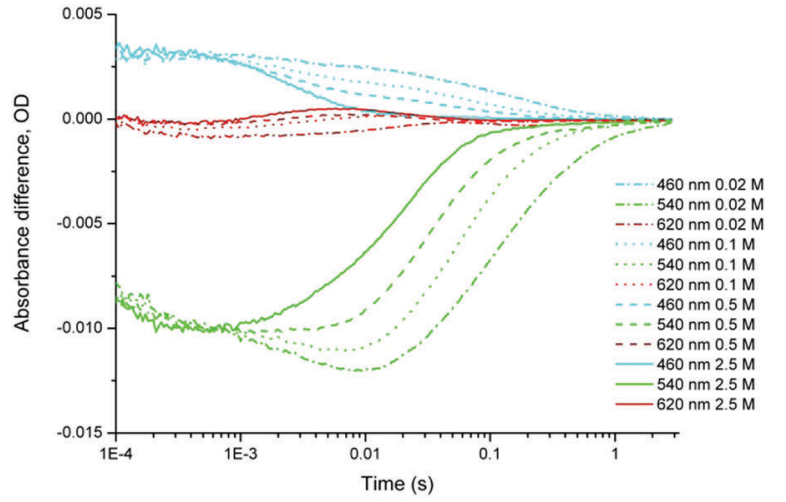

Fig. 2 Chloride dependence of the MastR photocycle in E. coli membranes encased in polyacrylamide gel, measured at $\mathrm{pH} 6$, buffered by $0.05 \mathrm{M} \mathrm{KH}_{2} \mathrm{PO}_{4}$ and $0.05 \mathrm{M} \mathrm{MES}$, at $22{ }^{\circ} \mathrm{C}$, over the range of $0.02-2.5 \mathrm{M} \mathrm{NaCl}$ concentrations. All traces are normalized based on $540 \mathrm{~nm}$ difference absorption amplitude at $0.7 \mathrm{~ms}$ time point.

Surprisingly, the photocycle turnover was very slow $(\sim 6 \mathrm{~s}$ lifetime), from which it was argued that MrHR is not an efficient chloride pump. ${ }^{44}$ While detergent-solubilized rhodopsins provide optically superior samples because of low scattering, removal of lipids often changes the photocycle kinetics and ion affinities of some functional groups. For this reason, we opted for characterizing the photocycle of MastR and its chloride dependence in a presumably more native system, E. coli membrane fragments. Even though this system does not allow for the detection of the early steps of the photocycle (faster than $0.1 \mathrm{~ms}$ ) due to relatively high scattering, it permits the study of the late steps relevant for chloride transport events in the lipid environment. Fig. 2 shows that the photocycle of MastR in lipid bilayers has much faster turnover than that reported in detergent (lifetime of about $0.3 \mathrm{~s}$ in membranes compared to $6 \mathrm{~s}$ in detergent at $100 \mathrm{mM} \mathrm{NaCl}$ ). This suggests that the ion pumping efficiency of MastR may be higher than previously thought, even though it is still significantly lower than those of other chloride-pumping microbial rhodopsins (as photocycles of HRs and ClRs have typical lifetimes of $0.02-0.03 \mathrm{~s}$ ). ${ }^{36,37,40,43,54}$

Flash photolysis kinetic traces of MastR-containing E. coli membranes exhibit a clearly chloride-dependent photocycle (Fig. 2 and 3), characteristic of chloride pumping rhodopsins. It should be noted that we explored chloride-dependence of the late photocycle steps at the anion concentrations well above the chloride affinity in the dark, to avoid contamination by the nontransporting (chloride-free) photocycle. Chloride affinity of the dark state in detergent was reported to be around $2 \mathrm{mM}^{44}$ and we verified this value for membrane-embedded MastR by titrating the amplitude of the $\mathrm{L} / \mathrm{N}$ intermediates, which are observed at $460 \mathrm{~nm}$ only in the presence of chloride ions (Fig. S4, ESI $\dagger$ ). The titration gave us the chloride affinity of the dark state of MastR of $\sim 2 \mathrm{mM}$, ensuring that the lowest concentration of chloride used in the study of the chloride-dependent photocycle kinetics $(20 \mathrm{mM})$ is well above the saturation.

Similar to what was observed for MastR in detergent, ${ }^{44}$ we measured the $\mathrm{L}$ and $\mathrm{N}$ intermediates signal at $460 \mathrm{~nm}$, the $\mathrm{O}$ 

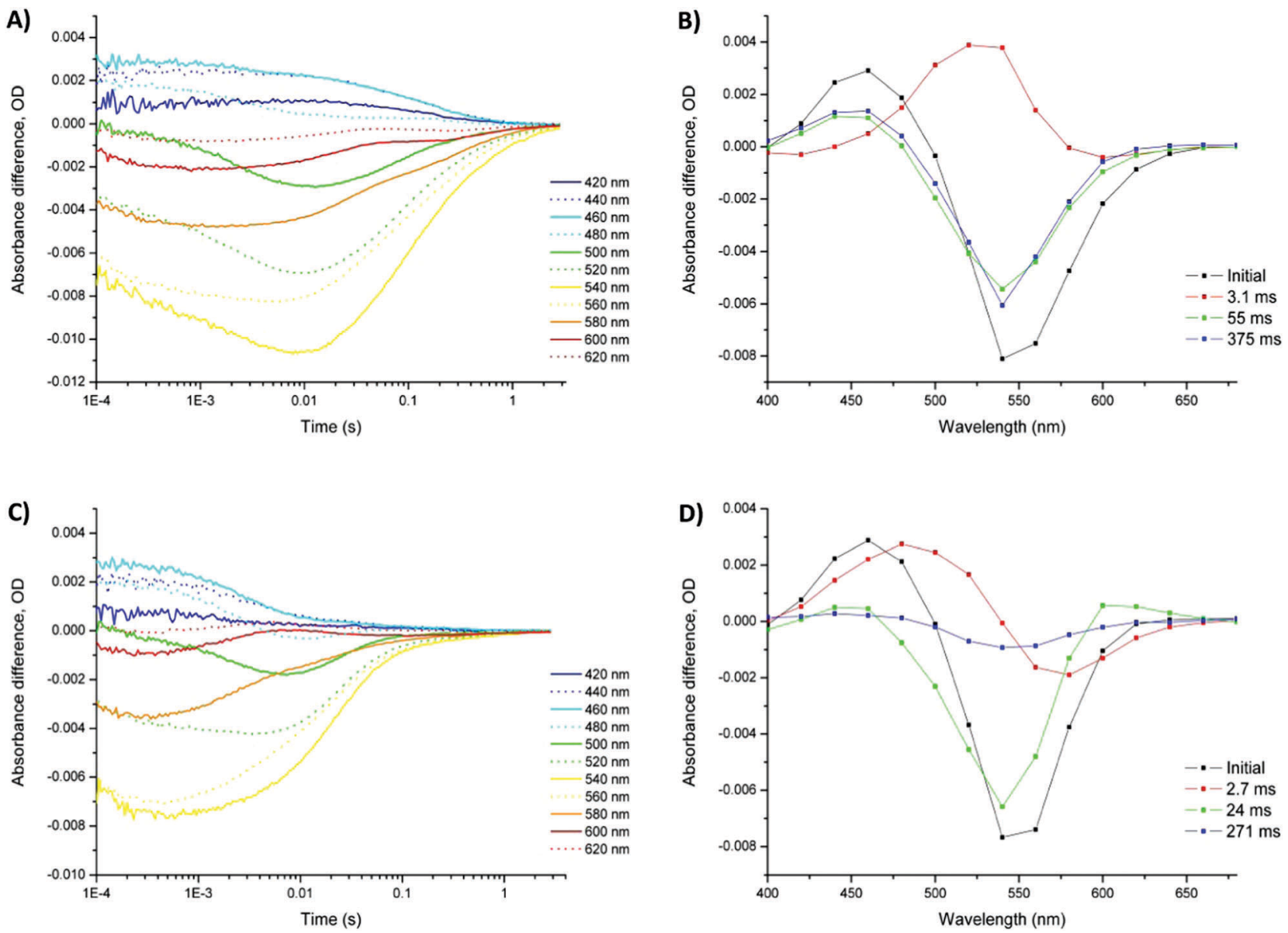

Fig. 3 Wavelength scans (the $400 \mathrm{~nm}, 640 \mathrm{~nm}, 660 \mathrm{~nm}$, and $680 \mathrm{~nm}$ traces were omitted for clarity) of the MastR photocycle in E. coli membranes encased in polyacrylamide gels at pH 6 in $0.02 \mathrm{M} \mathrm{NaCl}(\mathrm{A})$ and $2.5 \mathrm{M} \mathrm{NaCl}(\mathrm{C})$, with other conditions as in Fig. 2. Spectra of the kinetic components of the MastR photocycle extracted by global multi-exponential analysis for $0.02 \mathrm{M} \mathrm{NaCl}(\mathrm{B})$ and $2.5 \mathrm{M} \mathrm{NaCl}(\mathrm{D})$. The spectrum labelled "Initial" represents a lightminus-dark difference spectrum at the beginning of the fit ( $300 \mu \mathrm{s})$. Characteristic times of the spectral components are shown in the legend and discussed in the text. In the decay-associated spectra, the negative absorption means formation of intermediates, whereas the positive absorption corresponds to their disappearance.

intermediate at $620 \mathrm{~nm}$, and another ground state-like intermediate, MastR' (detected as a disappearance of the dark state) at $540 \mathrm{~nm}$ (Fig. 2 and 3). The presence of these intermediates is typical for most chloride pumping rhodopsins, however, it appears that the order of molecular events may be distinct from that in haloarchaeal chloride pumps such as pHR. In contrast to $\mathrm{pHR},{ }^{36,55,56}$ the $\mathrm{O}$ intermediate accumulates at high concentrations of chloride but not at the low ones, where it is replaced by the $\mathrm{N}$ intermediate, observed as additional bleaching of the dark state and persisting positive signal at $460 \mathrm{~nm}$ (Fig. 2). Thus, at low chloride concentrations the late part of the photocycle is dominated by the $\mathrm{N}$ intermediate (positive signal at $460 \mathrm{~nm}$ and growth of the negative signal at $540 \mathrm{~nm}$ ), while at high chloride concentrations we observe more $\mathrm{O}$ intermediate (at $620 \mathrm{~nm}$ ), followed by the Mast ${ }^{\prime}$ intermediate (no positive signal at $460 \mathrm{~nm}$ in the presence of negative signal at $540 \mathrm{~nm}$ ). This sequence of events is supported by the global multi-exponential analysis of the full wavelength scans (taken every $20 \mathrm{~nm}$ between 400 and $680 \mathrm{~nm}$ ) that were performed at $0.02 \mathrm{M}$ and $2.5 \mathrm{M} \mathrm{NaCl}$ (Fig. 3).
Fig. $3 \mathrm{~B}$ and $\mathrm{D}$ show the initial light-induced difference spectra which represent a "snapshot" at $\sim 300 \mu$ s along with the ensuing decay-associated spectra gleaned from threeexponential fits of the data in Fig. 3A and C. The "Initial" difference spectra at both chloride concentrations show formation of the $\mathrm{L}$ intermediate with the typical maximum of about $470 \mathrm{~nm}$ and the minimum of about $540 \mathrm{~nm}$, which is close to the absorption maximum of the dark state. The later decayassociated spectra are difference spectra corresponding to the specified kinetic components, where positive peaks correspond to disappearance and negative peaks correspond to formation of intermediate(s) and/or their mixtures. Beginning with $0.02 \mathrm{M}$ $\mathrm{NaCl}$ (Fig. 3B), the $3.1 \mathrm{~ms}$ component shows what can be interpreted as the $\mathrm{L}$ to $\mathrm{N}$ state transition without significant accumulation of the $\mathrm{O}$ intermediate. The next two kinetic components, with characteristic times of $55 \mathrm{~ms}$ and $375 \mathrm{~ms}$, have similar spectra showing the $\mathrm{N}$ intermediate decay. Non mono-exponential decay of the $\mathrm{N}$ intermediate suggests that it occurs through another intermediate, possibly MastR', which can be clearly observed at higher chloride concentrations only. 
For the $2.5 \mathrm{M} \mathrm{NaCl}$ data (Fig. 3D), starting from the similar initial spectrum showing formation of the $\mathrm{L}$ state, one can observe clear formation of the $\mathrm{O}$ intermediate (negative peak at $580 \mathrm{~nm}$, likely mixed with $\mathrm{N}$ ) with the characteristic time of $2.7 \mathrm{~ms}$. The component with $24 \mathrm{~ms}$ lifetime shows that the N/O intermediate mixture decays into the dark state-like MastR' intermediate. Finally, the last minor component with $271 \mathrm{~ms}$ lifetime shows the decay of the MastR' intermediate back to the dark state.

One of the simplest ways to explain the observed chloride dependence is to assume that, similar to $\mathrm{pHR},{ }^{37,57}$ the $\mathrm{L}$ intermediate decay (in about $3 \mathrm{~ms}$ ) produces an equilibrium mixture of the $\mathrm{N}$ and $\mathrm{O}$ states and that this equilibrium is chloride-dependent. This chloride-dependence suggests that the chloride uptake occurs during $\mathrm{O}$ intermediate formation, rather than during its decay, contrary to what happens in pHR. $^{36}$ Thus, at low chloride concentrations, chloride uptake becomes rate limiting in the equilibrium between $\mathrm{N}$ and $\mathrm{O}$ and shifts the equilibrium back towards $\mathrm{N}$, so that a long-lived $\mathrm{N}$ intermediate is observed and no $\mathrm{O}$ (or MastR') intermediate accumulates. At high chloride concentrations, chloride uptake is no longer rate limiting and the equilibrium is shifted toward the $\mathrm{O}$ intermediate. As a result, the $\mathrm{N}$ intermediate decays quickly without significant accumulation, but the $\mathrm{O}$ intermediate is accumulated. Under these conditions, its rise reports on the $\mathrm{L} \rightarrow \mathrm{N}$ transition, and its relatively fast decay allows the Mast ${ }^{\prime}$ intermediate to be observed. This situation can be visualized by the following preliminary scheme of the late part of the photocycle as based on the flash-photolysis data in $E$. coli membranes (the characteristic apparent time constants are given for $2.5 \mathrm{M}$ $\mathrm{NaCl}$ ), which will be refined further based on the flash-photolysis data in synthetic lipids, infrared spectroscopy and mutagenesis data presented below.

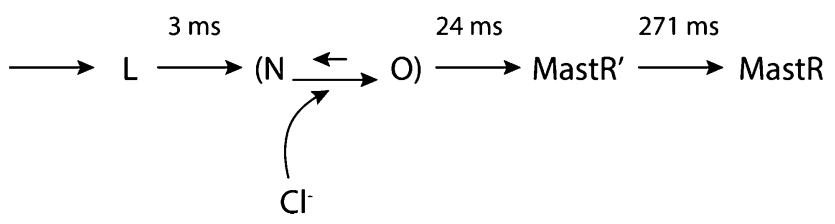

To get further insights into the molecular mechanism of anion transport by MastR using vibrational spectroscopy, we had to purify the protein and reconstitute it into synthetic lipids at high protein/lipid ratio (see Experimental above). Prior to performing FTIR and Raman spectroscopies, a control flashphotolysis experiment was conducted on the MastR proteoliposome films at high chloride concentration $(\sim 3$ M, Fig. S5, ESI $\dagger)$ to ensure that the kinetic behaviour of this lipid-reconstituted sample was comparable to that of the native-like in-membrane sample shown above (Fig. 2). Overall, the kinetics of the late photocycle intermediates (the L, N, O, and MastR' states) were found to be comparable to those in $E$. coli membranes. In proteoliposomes, the MastR' intermediate is observed more clearly after the $\mathrm{L}, \mathrm{N}$, and $\mathrm{O}$ intermediates have decayed completely. With the improved protein density and optical properties of the purified sample, the temporal resolution was enhanced so that the tail of the $\mathrm{K}$ intermediate decay could be observed in the microsecond range (at $620 \mathrm{~nm}$ ). Additionally, a multi-exponential character of the $\mathrm{L}$ intermediate rise (at $460 \mathrm{~nm}$ ) could be detected, arguing for the existence of at least two L intermediates, similar to $\mathrm{pHR},{ }^{37,56,58}$ with the $\mathrm{L} 1$ state forming in a few microseconds and the L2 state in $\sim 60 \mu \mathrm{s}$.

\subsection{Characterization of the MastR photocycle by vibrational spectroscopy}

3.2.1 Raman spectroscopy of the dark state of MastR. Prior to the time-resolved FTIR measurements, the retinal conformation and hydrogen-bonding in the dark state of MastR were investigated using Raman spectroscopy (Fig. 4). Overall, the spectra are very similar to those reported for the close homolog of MastR, SyHR. ${ }^{45}$ The position of the main ethylenic $\mathrm{C}=\mathrm{C}$ stretch band $\left(1534 \mathrm{~cm}^{-1}\right)$ is consistent with the colour of $E$. coli expressed MastR, ${ }^{59}$ whose absorption maximum in lipid-reconstituted samples is $534 \mathrm{~nm}$ (data not shown). The main fingerprint C-C stretching vibrations at 1200 and $1165 \mathrm{~cm}^{-1}$ and the virtual absence of the $1184 \mathrm{~cm}^{-1}$ band indicate that retinal is predominantly in the all-trans configuration, ${ }^{60}$ without any appreciable dark- or light-adaptation (not shown), consistent with the earlier retinal extraction data. ${ }^{44}$ This ensures that we will be probing only the photocycle of the all-trans-MastR.

To probe hydrogen-bonding strength of the retinal Schiff base we followed the isotopic shift of the $\mathrm{C}=\mathrm{N}$ stretching band, ${ }^{61}$ which occurs at $1639 \mathrm{~cm}^{-1}$ in $\mathrm{H}_{2} \mathrm{O}$ and at $1623 \mathrm{~cm}^{-1}$ in $\mathrm{D}_{2} \mathrm{O}$ (with corresponding $\mathrm{N}-\mathrm{D}$ wags coming up at $977 \mathrm{~cm}^{-1}$ ). In similarity to the homologous SyHR, ${ }^{45}$ the magnitude of this isotopic shift is the same as in $\mathrm{BR}\left(\sim 16 \mathrm{~cm}^{-1}\right),{ }^{62}$ but somewhat larger than what was observed in other chloride pumps, HRs and ClRs, as well as the chloride-transporting BR mutant D85T (up to $12 \mathrm{~cm}^{-1}$ ). ${ }^{63-66}$ This result confirms somewhat stronger hydrogen bonding of the Schiff base in cyanobacterial anion pumps compared to other chloride pumps, ${ }^{45}$ which may be consistent with the more BR-like environment of the Schiff base

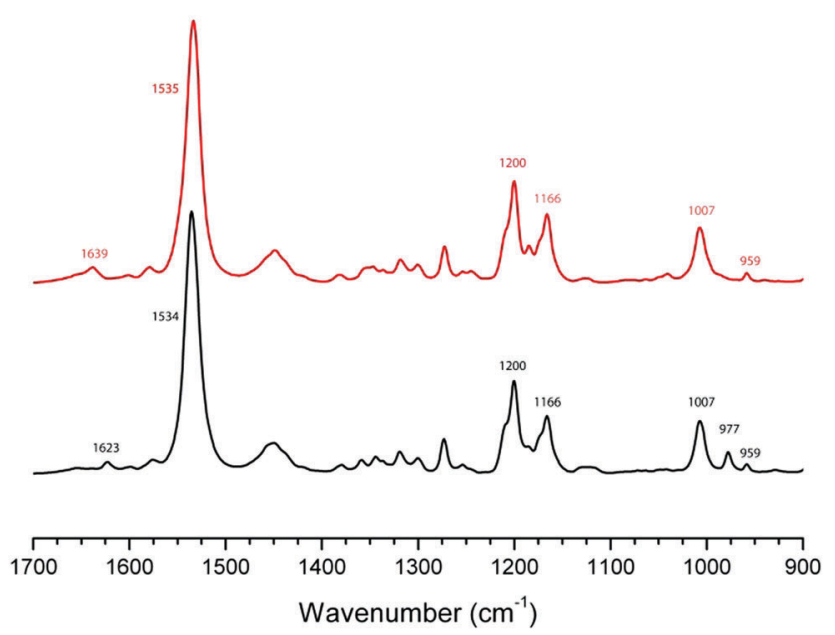

Fig. 4 Raman spectra of dark-adapted lipid-reconstituted MastR in $0.5 \mathrm{M}$ $\mathrm{NaCl}, 0.05 \mathrm{M} \mathrm{MES}$ and $0.05 \mathrm{M} \mathrm{KH}_{2} \mathrm{PO}_{4}$ at $\mathrm{pH} 6$ (shown in red), and in the same $\mathrm{D}_{2} \mathrm{O}$-based buffer at pD 6 (shown in black). The spectra are normalized by the ethylenic stretch amplitude. 


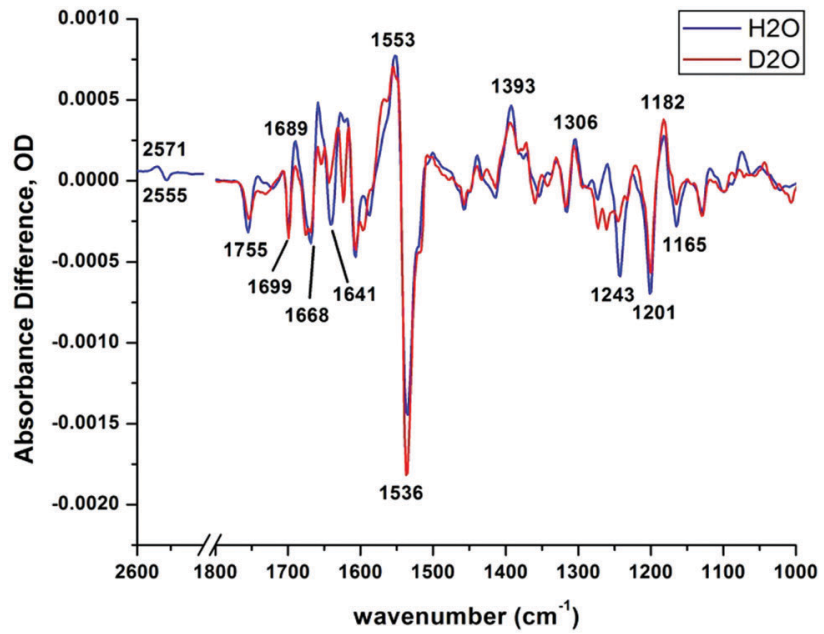

Fig. 5 Light-minus-dark difference FTIR spectra of MastR proteoliposomes (sample conditions as in Fig. S5, ESI $\dagger$ ), in $\mathrm{H}_{2} \mathrm{O}$ (blue) and $\mathrm{D}_{2} \mathrm{O}$ (red) based buffer, under steady-state green $(530 \mathrm{~nm}$ ) illumination ( $2 \mathrm{~s}$ on minus $2 \mathrm{~s}$ off, 50 cycles average). Spectral resolution is $4 \mathrm{~cm}^{-1}$.

in this group. We observed a similar shift of the Schiff base $\mathrm{C}=\mathrm{N}$ vibrations (from $1641 \mathrm{~cm}^{-1}$ in $\mathrm{H}_{2} \mathrm{O}$ to $1624 \mathrm{~cm}^{-1}$ in $\mathrm{D}_{2} \mathrm{O}$ ) in the FTIR data (Fig. 5).

3.2.2 FTIR spectroscopy of the MastR' intermediate. The photocycle kinetics of MastR in synthetic lipids (Fig. S5, ESI $\dagger$ ) show very clear separation of the last photocycle intermediate (MastR ${ }^{\prime}$ ) from the rest of the late intermediates in the $30 \mathrm{~ms}-1 \mathrm{~s}$ time range. This allowed us to probe molecular details of MastR' with steady-state difference FTIR spectroscopy, where it accumulates under quasi-continuous ( $2 \mathrm{~s}$ on/2 s off) $530 \mathrm{~nm}$ LED illumination (Fig. 5). It should be noted that some late intermediates in the photocycles of microbial rhodopsins show pronounced secondary photochemistry, ${ }^{67-69}$ which may make any conclusions based on the studies of photostationary mixtures somewhat tentative. To avoid this problem, we verified our steady-state results by time-resolved rapid-scan FTIR difference spectroscopy with $\sim 30 \mathrm{~ms}$ time resolution (Fig. S6, ESI $\dagger$ ), which showed the spectra very similar to those obtained under continuous illumination.

The MastR'-minus-MastR difference spectra (Fig. 5 and Fig. S6, ESI $\dagger$ ) yield a number of interesting insights into molecular properties of the last photocycle intermediate as well as of the dark state. First, the $\mathrm{C}-\mathrm{C}$ stretching fingerprint region shows strong negative bands at 1243, 1201, and $1165 \mathrm{~cm}^{-1}$, along with the positive band at $1182 \mathrm{~cm}^{-1}$, with the 1243 and $1165 \mathrm{~cm}^{-1}$ bands being partially shifted in $\mathrm{D}_{2} \mathrm{O}$. This fingerprint pattern is much more similar to those observed in the photointermediates of BR with 13-cis-protonated Schiff base ( $\mathrm{L}$ and $\mathrm{N}$ ) than in the respective intermediates of $\mathrm{SHR}$ and $\mathrm{pHR}$, where a strong negative band is observed around $1210 \mathrm{~cm}^{-1}$ and BR's prominent $1254 \mathrm{~cm}^{-1}$ band (a counterpart of the $1243 \mathrm{~cm}^{-1}$ in MastR and several other microbial rhodopsins) is strongly reduced. ${ }^{5,56,70,71}$ This once again suggests more BR-like (rather than HR-like) character of the retinal-binding pocket of MastR, which is also consistent with the conservation pattern highlighted in Fig. S1, ESI. $\dagger$ A number of residues in the vicinity of retinal (or known to affect it via long-range interactions in the hydrogen-bonded networks) are common between MastR and BR, but not HR, including BR's A53, Y79, E204, V217, and G220. Among these, Ala-53 mutations are known to have a strong effect on retinal Schiff base $\mathrm{p} K_{\mathrm{a}}$ and water binding. ${ }^{72}$

Another surprising fact which can be deduced from the fingerprints pattern is that the Mast $\mathrm{R}^{\prime}$ intermediate contains 13-cis-retinal, suggesting that its thermal re-isomerization occurs in the last step of the photocycle. This is not what would be expected for an intermediate which follows the $\mathrm{O}$ state, known to contain all-trans-retinal in $\mathrm{pHR}$ and $\mathrm{BR},{ }^{56,73}$ even though red-shifted O-like states with 13-cis-retinal have been observed in some microbial rhodopsins. ${ }^{74,75}$

In addition to the BR-like 13-cis-retinal fingerprint pattern discussed above, the Schiff base vibrations at $1641 \mathrm{~cm}^{-1}$ and possible amide I perturbation at $1668 \mathrm{~cm}^{-1}$ (similar to that of the $\mathrm{N}$ intermediate of $\mathrm{BR})^{76}$ in the difference spectrum of MastR' suggest a significant conformational change of this state compared to the dark state. This idea is confirmed by the appearance of several unusual bands showing perturbation of polar sidechains, including cysteine, carboxylic acids, and possibly arginine and asparagine. The cysteine perturbation is observed at $2555(-) / 2571(+) \mathrm{cm}^{-1}$, and a cysteine residue responsible for this feature was found to be not H/D exchangeable judging from the absence of typical S-D vibrations around $1860 \mathrm{~cm}^{-1}$ (not shown). A similar non-exchangeable buried Cys residue was observed in the late intermediates of the Neurospora rhodopsin photocycle, ${ }^{77}$ and it is tempting to assign this vibration in both proteins to the cysteine located near the $\beta$-ionone ring of retinal (position 142 using BR numbering) as this residue is present in MastR as well (Cys-130). On the other hand, it should be noted that the MastR sequence contains two more plausible candidates for this band, Cys-43, which is expected to be located in the chloride release channel, and Cys-77, which is close to the chloride-coordinating motif-forming Thr-74 and Ser-78 (see Fig. S1, ESI $\dagger$ ).

A pair of bands which could originate from perturbation of sidechains of Arg/Asn residues is prominent at $1699(-) /$ $1689(+) \mathrm{cm}^{-1}$, with the positive band being $\mathrm{D}_{2} \mathrm{O}$-dependent, and an additional weak positive band at $1706 \mathrm{~cm}^{-1}$. Similar major bands were observed in sHR and pHR, and tentatively assigned to the superconserved homolog of BR's Arg-82, ${ }^{78-80}$ which is present in MastR as well (Arg-71). Additionally, MastR has two Asn residues expected to be in the conformational hotspots, which could contribute to this spectral region. First, Asn-39 is expected to be located in the chloride release channel (as a homolog of BR's Thr-46). Second, Asn-104 is a homolog of Asp-115 of BR, the residue known to be involved in strong interhelical hydrogen-bonding, which has prominent spectral signatures both for BR and HRs. ${ }^{71,80,81}$

Finally, likely the most interesting feature of the MastR' difference spectrum is a strong putative deprotonation band of a carboxylic acid located at $1755 \mathrm{~cm}^{-1}$ (with its positive counterpart likely to be at $1393 \mathrm{~cm}^{-1}$ ) (Fig. 5 and Fig. S6, ESI $\dagger$ ). Before we 
discuss the origin and implications of this band, we should note the presence of a pair of weak bands at $1722(-) / 1742(+) \mathrm{cm}^{-1}$. The former band was observed in pHR at $1721 \mathrm{~cm}^{-1}$ and assigned to transient deprotonation of Glu-234 (homolog of BR's Glu-194 present in MastR). ${ }^{80}$ Returning to the putative Asp deprotonation band at $1755 \mathrm{~cm}^{-1}$, we should note that there are only two internal carboxylic acids in MastR, Asp-200 (homolog of BR's Asp-212) and the motif-forming Asp-85 (homolog of BR's Asp-96). As Asp-200 is expected to be part of the Schiff base counterion, it is unlikely to be protonated in the dark state, leaving Asp-85 as the most likely candidate. It is reasonable to assume that Asp-85 is protonated in the dark just like its homolog Asp-96 of BR, as most of its hydrophobic environment in the cytoplasmic ends of helices B, C, F, and G is conserved (Fig. S1, ESI $\dagger$ ). A confounding issue precluding this rather straightforward assignment is an untypically small deuteriuminduced shift of this band, which amounts to only $2 \mathrm{~cm}^{-1}$ (Fig. 5). Such small isotopic shifts were observed in some cases and interpreted as strong hydrogen-bonding of the $\mathrm{OH}$ group of the respective aspartic acid. ${ }^{81-83}$ To make a reliable assignment, we repeated steady-state FTIR measurements of MastR' spectra with the D85I mutant of MastR (Fig. 6). Indeed, the $1755 \mathrm{~cm}^{-1}$ band of a protonated carboxylic acid has disappeared as expected while the corresponding carboxylate band at $1393 \mathrm{~cm}^{-1}$ got dramatically reduced. This allows us to assign the $1755 \mathrm{~cm}^{-1}$ band to the protonated carboxylic stretch of Asp-85 in the dark state of MastR and to claim that its hydroxyl group is strongly hydrogen-bonded and gets transiently deprotonated during the photocycle. The small downshift observed upon the H/D exchange of Asp-85 is in contrast to the much larger shift observed for its homolog Asp-96 in BR ( 2 vs. $\left.9 \mathrm{~cm}^{-1}\right),{ }^{81}$ suggesting much stronger hydrogenbonding in MastR. This can be tentatively explained by a different pattern of interhelical hydrogen-bonding between

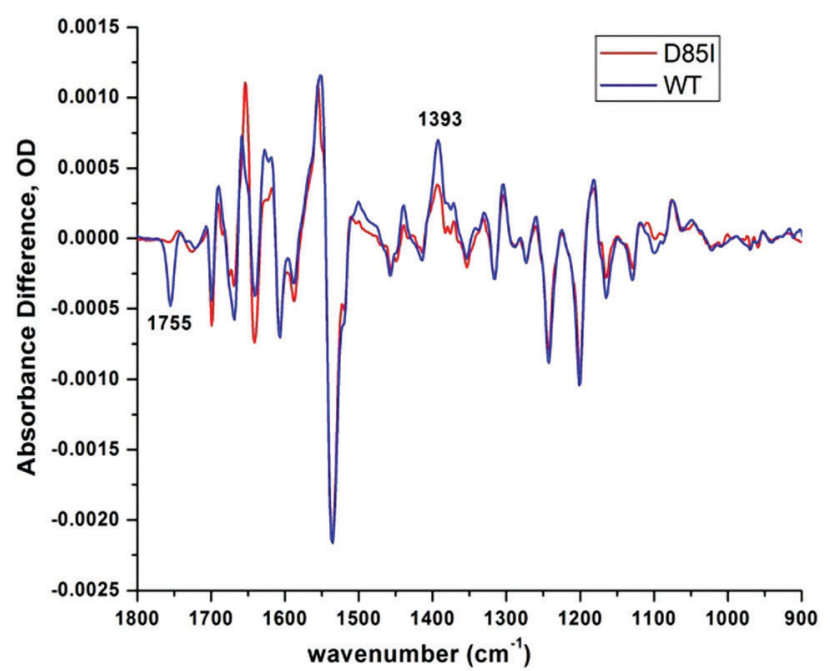

Fig. 6 Light-minus-dark difference FTIR spectra of MastR proteoliposomes (sample conditions as in Fig. S5, ESI ) , for the wild-type (blue) and D85I mutant (red), under steady-state green $(530 \mathrm{~nm}$ ) illumination (2 s on minus $2 \mathrm{~s}$ off, 50 cycles average). Spectral resolution is $4 \mathrm{~cm}^{-1}$. The wildtype spectrum is taken from Fig. 5 for comparison, with the multiplication factor of 1.5 . the two proteins. In BR, Asp-96 interacts with Thr-46, while in MastR Asp-85 may interact with homologous Asn-39, which can provide stronger hydrogen-bonding compared to the threonine. Indeed, the $1755 \mathrm{~cm}^{-1}$ band downshifts in the N39A mutant to $1750 \mathrm{~cm}^{-1}$ (not shown), confirming our hypothesis of Asp-85/ Asn-39 interaction. Interestingly, the position of BR's Asp-96 vibration in the T46V background is virtually the same as that of MastR's Asp-85 in the N39A background, ${ }^{84}$ suggesting that in the absence of the interhelical hydrogen-bonding the environment of Asp-85 is very close to that of its homolog in BR.

3.2.3 FTIR spectroscopy of the earlier photocycle intermediates. To get further insights into the molecular events in the photocycle of MastR and to reveal kinetics of formation of the bands detected in the Mast $\mathrm{R}^{\prime}$ intermediate (see above), the earlier intermediates were explored by step-scan FTIR spectroscopy with $6.25 \mu$ s time resolution. Fig. 7 shows three characteristic time slices of this dataset, which are expected to represent the L1 (at $6.25 \mu \mathrm{s}$ ), L2 (at $203 \mu \mathrm{s}$ ), and N/O (at $4.47 \mathrm{~ms}$ ) intermediates, based on the flash-photolysis data (Fig. S5, ESI $\dagger$ ). The earliest spectrum (L1) shows L-like fingerprint vibrations with the positive band at $1189 \mathrm{~cm}^{-1}$, which shifts to lower (N-like) positions in the later intermediates ( $\mathrm{L} 2$ and N/O), similar to the situation in BR. ${ }^{76,85,86}$ It also shows a prominent band at $1154 \mathrm{~cm}^{-1}$ known for the cryotrapped L intermediate of $\mathrm{BR} .{ }^{86}$ Perturbation of the Schiff base is obvious from the $1639 \mathrm{~cm}^{-1} \mathrm{C}=\mathrm{N}$ stretching band (shifts to $1623 \mathrm{~cm}^{-1}$ accompanied by the ND vibrations at $970 \mathrm{~cm}^{-1}$ in $\mathrm{D}_{2} \mathrm{O}$, not shown). It should be noted that, consistent with the expectations from the 13-cis-retinal configuration of Mast ${ }^{\prime}$, the spectra taken at the time of the maximal $\mathrm{O}$ accumulation do not show any typical features of the $\mathrm{O}$ intermediate of BR, such as positive all-trans-retinal fingerprints at

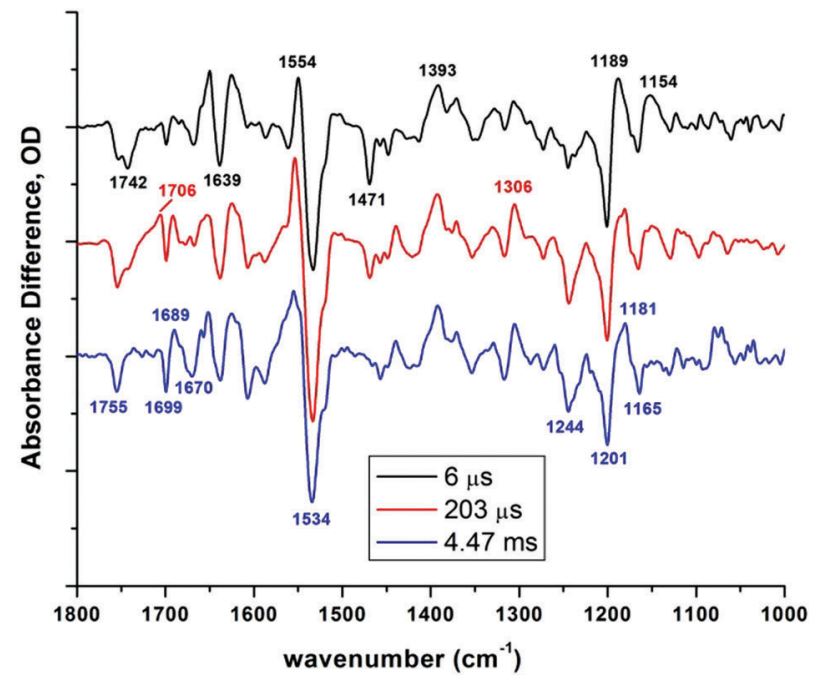

Fig. 7 Time slices of the SVD-filtered step-scan light-minus-dark difference FTIR spectra of MastR proteoliposomes (sample conditions as in Fig. S5, ESI $\dagger$ ) in the 1800-1000 $\mathrm{cm}^{-1}$ range, expected to represent the L1 (at $6 \mu \mathrm{s}$ ), L2 (at $203 \mu \mathrm{s}$ ), and N/O (at $4.47 \mathrm{~ms}$ ) intermediates. Time resolution is $6.25 \mu \mathrm{s}$, spectral resolution is $4 \mathrm{~cm}^{-1}$. Each spectrum is vertically offset by $0.002 \mathrm{OD}$ from the preceding one for clarity. Minor ticks on the vertical scale correspond to $0.001 \mathrm{OD}$. 
$1168 \mathrm{~cm}^{-1} \cdot{ }^{87}$ Even though the 13-cis-retinal configuration in the intermediates preceding Mast $\mathrm{R}^{\prime}$ is logical, one should be mindful of the fact that the $\mathrm{O}$ intermediate accumulation appears to be low, as judged from the visible spectroscopy data, so that its spectral signatures could be obscured by those of other intermediates. Another N-like signature, ${ }^{76}$ which develops on the time scale of transition from $\mathrm{L} 2$ to N/O and reports on the backbone conformational changes, possibly associated with chloride release, is the negative amide I band at $\sim 1670 \mathrm{~cm}^{-1}$ (Fig. 7 and 8 , also see the time evolution of this band in the lower noise $\mathrm{D}_{2} \mathrm{O}$ data in Fig. S7, ESI $\dagger$ ).

In the Arg/Asn region, several bands show interesting dynamics (Fig. 7). In the L1 intermediate only a weak negative band at $1699 \mathrm{~cm}^{-1}$ is present, while the strong doublet at $1699(-) / 1689(+) \mathrm{cm}^{-1}$ observed in MastR $^{\prime}$ (see Fig. 5 above) forms in $\mathrm{L} 2$ and persists until the end of the photocycle. On the other hand, a strong $1706 \mathrm{~cm}^{-1}$ band is seen only in the $\mathrm{L} 2$ intermediate, being absent in the $\mathrm{L} 1$ and N/O states. We tend to assign the $1699(-) / 1689(+) \mathrm{cm}^{-1}$ doublet to $\mathrm{Arg}-71$, based on its similarity to the bands observed in HR both at 170 and $250 \mathrm{~K}$ (early and late L states). ${ }^{71,80}$ It is likely that the $1706 \mathrm{~cm}^{-1}$ vibration originates from Asn-104 (homolog of Asp-115 of BR and Asp-134 of sHR). Perturbation of this residue is observed in sHR at $250 \mathrm{~K}$, but not at $170 \mathrm{~K}$, indicating a conformational change later in the photocycle, and a similar situation may exist in MastR, manifesting itself in the transient perturbation of the homologous Asn involved in interhelical hydrogen-bonding.

The protonated carboxylic acid region in the spectra of the earlier intermediates reports on the timing of deprotonation of

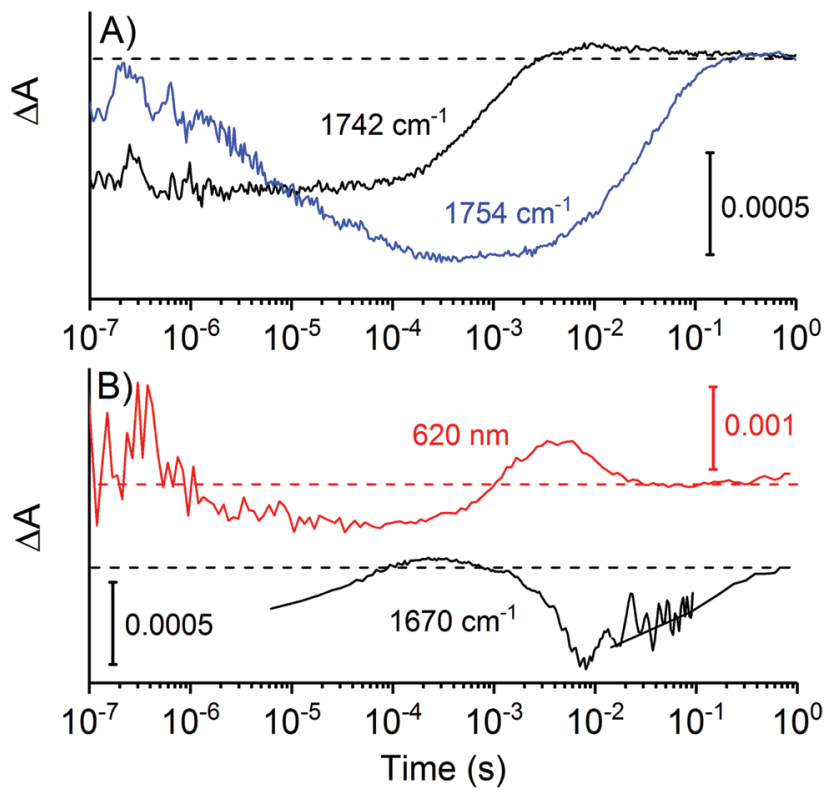

Fig. 8 Kinetics of light-induced absorption changes at selected wavenumbers, reporting on infrared signatures of protonated carboxylic acids (and/or lipid esters) (A) and protein backbone (amide I) (B). The kinetics at 1742 and $1755 \mathrm{~cm}^{-1}$ in (A) were measured using the QCL set-up (see Experimental), while the kinetics at $1670 \mathrm{~cm}^{-1}$ (black) in (B) were extracted from the step-scan (Fig. 7) and the rapid-scan (Fig. S6, ESI†) data, and compared to the flash-photolysis data at $620 \mathrm{~nm}$ (red) from Fig. S5 (ESI†).
Asp-85, which occurs between the $\mathrm{L} 1$ and $\mathrm{L} 2$ states on the tens of microseconds time scale, as judged by the appearance of the negative $1755 \mathrm{~cm}^{-1}$ band (Fig. 7 and 8). It should be noted that perturbation of the homologous Asp-96 in the L intermediate of BR produced a negative band which was originally interpreted as its deprotonation. ${ }^{81,85,88}$ The appearance of the clear deprotonated carboxylate vibrations counterpart at around $1393 \mathrm{~cm}^{-1}$ (as seen in MastR ${ }^{\prime}$ upon comparison of the wild-type and the D85I mutant, Fig. 6) is an additional indicator which can confirm the deprotonation, even though it may be complicated by strong contributions from retinal vibrations. ${ }^{81,88}$ Accordingly, we observe growth of a positive band at around $1393 \mathrm{~cm}^{-1}$ (sitting on top of larger retinal vibrations) on the time scale of the L1 $\rightarrow$ L2 transition, matching the development of the $1755 \mathrm{~cm}^{-1}$ band. Our data show that there is an additional negative band at $1742 \mathrm{~cm}^{-1}$, which is dominant in the $6 \mu \mathrm{s}$ spectrum (L1), seen as a strong shoulder of the $1755 \mathrm{~cm}^{-1}$ band in the $203 \mu \mathrm{s}$ spectrum, and disappears in the later spectra (Fig. 7 and 8). Based on its insensitivity to the H/D exchange (data not shown) one may assign the $1742 \mathrm{~cm}^{-1}$ band to vibrations of lipid esters, known to contribute to the signal in this area for other retinal proteins. ${ }^{89,90}$ Another band which may reflect lipid perturbation and is seen on the same time scale is located at $1471 \mathrm{~cm}^{-1}$ and can be assigned to fatty acid tails of lipids. Alternatively, this band may originate from an unknown protonated carboxylic acid, such as one of the members of the extracellular Glu pair (Glu-182/Glu-192, homologs of Glu-194/Glu-204 of BR).

Finally, exploration of the time course of appearance of a cysteine perturbation (seen in MastR' $^{\prime}$ spectra at 2555(-)/ $2571(+) \mathrm{cm}^{-1}$, Fig. 5) yielded an unexpected result. Fig. 9 shows the spectral region of interest, from which it is obvious that Cys perturbation is already present in the L1 state and becomes stronger in the later intermediates. It also shows a much stronger

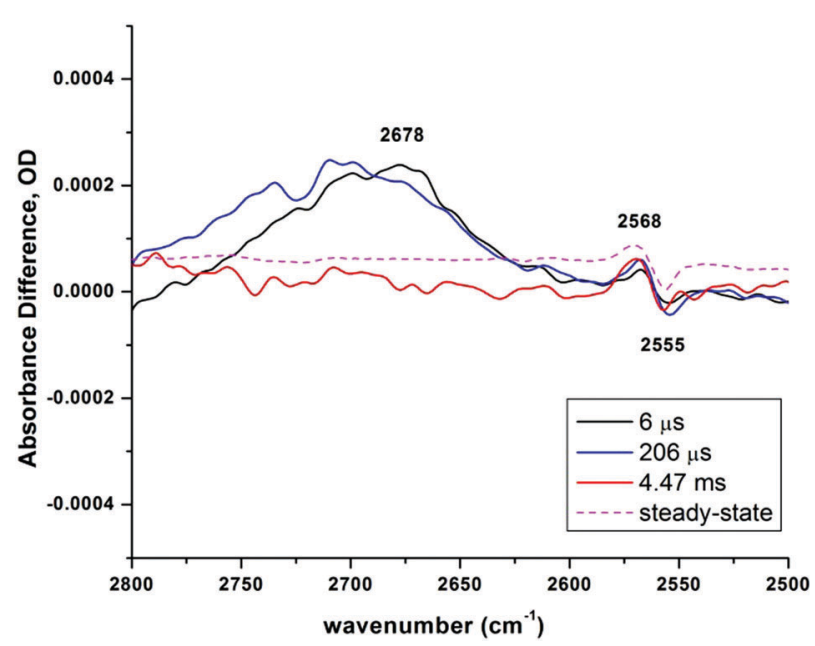

Fig. 9 Time slices of the SVD-filtered step-scan light-minus-dark difference FTIR spectra of MastR proteoliposomes (sample conditions as in Fig. S5, ESI $\dagger$ ) in the 2800-2500 $\mathrm{cm}^{-1}$ range, expected to represent the L1 (at $6 \mu \mathrm{s}$ ), L2 (at $206 \mu \mathrm{s}$ ), and N/O (at $4.47 \mathrm{~ms}$ ) intermediates. Time resolution is $6.25 \mu \mathrm{s}$, spectral resolution is $8 \mathrm{~cm}^{-1}$. The steady-state spectrum is reproduced from Fig. 5 for comparison. 
broad ("continuum") positive band centered at $2678 \mathrm{~cm}^{-1}$, which upshifts during the transition from L1 to L2 and disappears upon the $\mathrm{L} 2$ decay. This band is not observed in $\mathrm{D}_{2} \mathrm{O}$ (not shown) and is clearly distinct from the transient water heating signals observed earlier. ${ }^{91}$ Its position and linewidth are reminiscent of the spectral signatures of strongly hydrogen-bonded water clusters, including those of the Eigen cation. ${ }^{92-94}$ This implies that MastR experiences rearrangement of water molecules (transient clustering) in its L1 and L2 intermediates, similar to formation of the water clusters observed in the X-ray structure of the $\mathrm{N}$ intermediate of pHR in its cytoplasmic side, which may be important for chloride ion translocation. ${ }^{35}$

\subsection{Probing ion translocation pathways in the MastR photocycle by site-directed mutagenesis and modelling}

To further investigate molecular details of ion translocation by MastR we expressed a number of its mutants and studied their photocycles. The mutation sites (Fig. S1, ESI $\dagger$ ) were selected based on the amino acid conservation patterns in the new group of cyanobacterial anion pumps and on their comparison with those in other chloride transporters (HRs and ClRs) and in BR. Specifically, we targeted conserved intramembrane polar residues which were expected to be close to the putative ionconducting pathways as predicted from the MastR structural homology model (see Supplementary methods, ESI $\dagger$ and Fig. 10).

The structure of MastR obtained via homology modelling and subsequent optimization was used to inspect interactions of polar and charged groups that could be important for protein conformational dynamics and ion transport.

First, Asp-85 and Ser-211 could engage in an inter-helical hydrogen bond that resembles the Asp-96/Thr-46 pair in BR and inter-helical carboxylate/hydroxyl pairs identified in other membrane transporters $;^{95}$ in MastR, however, the dynamics
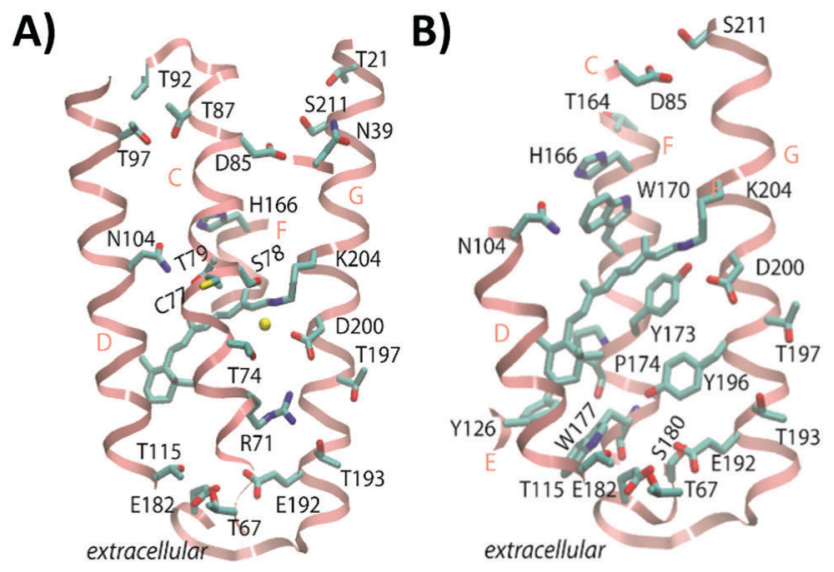

Fig. 10 Structural model of MastR with putative location of selected protein groups of MastR based on the geometry-optimized homology model. (A) A view focusing on helices $C, D$, and $G$; (B) a view focusing on helices $F$ and $G$. Selected protein segments are shown as pink ribbons, and retinal and selected protein sidechains are shown as sticks. The chloride ion is colored yellow, carbon atoms - cyan, oxygen - red, and nitrogen blue. The figure was prepared using the VMD software. ${ }^{1}$ at the Asp-85 site is likely more complex than in bR, because Asn-39 could participate in inter-helical hydrogen bonding, as also suggested by the FTIR data presented above. The Asn-39/ Asp-85/Ser-S211 cluster of MastR (Fig. 10) is reminiscent of the central Glu-Asn-Thr cluster of the SecY protein translocon, whose complex dynamics with breaking and forming of hydrogen bonds has been documented by computer simulations. ${ }^{96,97}$ In the case of MastR, breaking and forming of inter-helical hydrogen bonds would associate with changes in the protonation state of Asp-85.

Next, inspection of the MastR structural model indicates that some of the transmembrane segments include Ser/Thr groups that could have important roles in shaping the local structure and dynamics of the protein. We think that two clusters of Ser/Thr groups could be particularly important for the conformational dynamics of MastR. The first Ser/Thr cluster is located at the heart of the protein, where helix $\mathrm{C}$ contains three Ser/Thr and a Cys group (Fig. 10A); because Ser/Thr hydroxyl groups compete with backbone carbonyl groups for hydrogen bonding, ${ }^{98,99}$ their presence can lead to enhanced local dynamics of the helix. ${ }^{100}$ Local plasticity of helix $\mathrm{C}$ could facilitate chloride transport. The second cluster of Ser/Thr groups is at the cytoplasmic side of helices C and D, and it includes Thr-87, Thr-92, Ser-93, Thr-97, and Ser-99 (Fig. 10). The sequence alignments of the cyanobacterial anion pumps (Fig. S1, ESI $\dagger$ ) indicate significant conservation as Ser/ Thr for Ser-93, Thr-97, and Ser-99, whereas Thr-87 is present as Ser/Thr in almost all sequences analyzed here. These Ser/Thr hydroxyl groups can help couple the dynamics of helices $\mathrm{C}$ and $\mathrm{D}$, and provide a binding spot for the chloride ion. Since Asp-85 and Thr-87 are located within one helical turn of each other, protonation-coupled changes at the Asp-85 cluster could couple to the dynamics of chloride binding at the Thr-87 cluster. At the same time, it should be mentioned that sidechains of some of the above mentioned residues are predicted to be located away from the main ion-conducting path, likely to be formed by helices $\mathrm{B}, \mathrm{C}, \mathrm{F}$, and $\mathrm{G}$ as in other ion-pumping microbial rhodopsins, so that their exact role is hard to predict.

Finally, according to the homology model, His-166 on helix F could make $\pi$-stacking interactions with Trp-170, which is close to the $\mathrm{C}_{13}$ methyl group of the retinal polyene chain (Fig. 10B). These contacts could provide an elegant mechanism to couple the dynamics of His-166 to the dynamics and isomeric state of the retinal chromophore.

We began by mutating the TSD motif-forming Thr-74 and Ser-78, whose homologs in HR are involved in chloride binding in the dark. ${ }^{26-29}$ Similar to the situation observed for pHR, Ser-78 seems to be more important for chloride binding and protein stability than Thr-74..$^{29,38,101}$ The S78A MastR mutant displays a very significant decrease in the Schiff base $\mathrm{p} K_{\mathrm{a}}$ and stability, and bleaches even in the presence of chloride (not shown), similar to some of the Ser-130 mutants of pHR. ${ }^{101}$ When the Schiff base was stabilized by low $\mathrm{pH}(\mathrm{pH} 4)$, DDM-solubilized S78A MastR showed a large red shift of its absorption spectrum maximum (from $\sim 535 \mathrm{~nm}$ for the wild-type to $\sim 559 \mathrm{~nm}$ for the mutant, not shown), once again paralleling 
the behaviour of the S130A mutant of pHR. ${ }^{101}$ As the S78A protein was not stable under the neutral $\mathrm{pH}$ conditions, we have not studied its photocycle further. The T74A mutant also showed a red shift in the chloride-free DDM-solubilized form (from $\sim 535 \mathrm{~nm}$ for the wild-type to $\sim 548 \mathrm{~nm}$ for the mutant, not shown), similar to the behaviour of the homologous T111V mutant of sHR, but opposite to the T126V mutant of pHR. ${ }^{28,29}$ Similar to both HRs, it showed dramatically reduced chloride affinity in the DDM-solubilized state $\left(K_{\mathrm{d}}\right.$ of $\sim 85 \mathrm{mM}$ as opposed to the reported $2 \mathrm{mM}$ for the wild-type). ${ }^{44}$ At the same time, the absorption maximum and the photocycle of the chloride-bound form of the T74A mutant were not dramatically different from the wild-type, with an exception of the lack of observable accumulation of the $\mathrm{O}$ intermediate in the mutant (Fig. 11A). Considering much lower chloride affinity of the dark state of the T74A mutant this result may reflect a chloride ion location which differs from that in the wild-type $O$ intermediate. On the other hand, as the photocycle kinetics are not affected otherwise and do not resemble the wild-type kinetics at low chloride concentrations, one could argue that the chloride uptake is either unaffected or is not rate-limiting.

Next, we explored three conserved polar residues on the extracellular side of MastR, which could be conceivably involved in regulation of chloride transport, Glu-192, Thr-193, and
Thr-197 (Fig. S1, ESI $\dagger$ ). Thr-193 and Thr-197 are located on the same helical face (helix G, Fig. 10), and are conserved in HRs, suggesting their possible involvement in the chloride uptake pathway. Nevertheless, T193A and T197A mutants displayed virtually wild-type-like photocycles (Fig. 11) suggesting that these threonines do not play any major role in chloride conductance, even though one should keep in mind that chloride ions tend to form multiple hydrogen bonds so that single mutations do not always have strong effects on their coordination. Glu-192 is not conserved in HRs, but is a homolog of Glu-204 of BR, whose protonation state was shown to greatly affect chloride transport in the D85T mutant of BR via an electrostatic coupling. ${ }^{102}$ Replacement of Glu-204 in the chloride-transporting BR mutant (D85T/E204Q) resulted in dramatic acceleration of the O intermediate decay at neutral $\mathrm{pH}$, which was interpreted as a coupling of reprotonation of Glu-204 with chloride uptake on the extracellular side. The E192A mutant of MastR does not show the same behaviour, displaying a shift of the N/O equilibrium towards $\mathrm{N}$ and an accompanying delay of the photocycle turnover (Fig. 11B). This could be interpreted as a lack of transient deprotonation of Glu-192 in the photocycle of MastR along with participation of the protonated Glu-192 sidechain in chloride coordination during its uptake.

Lastly, we investigated the roles of a number of polar residues on the cytoplasmic side of MastR: the motif-forming
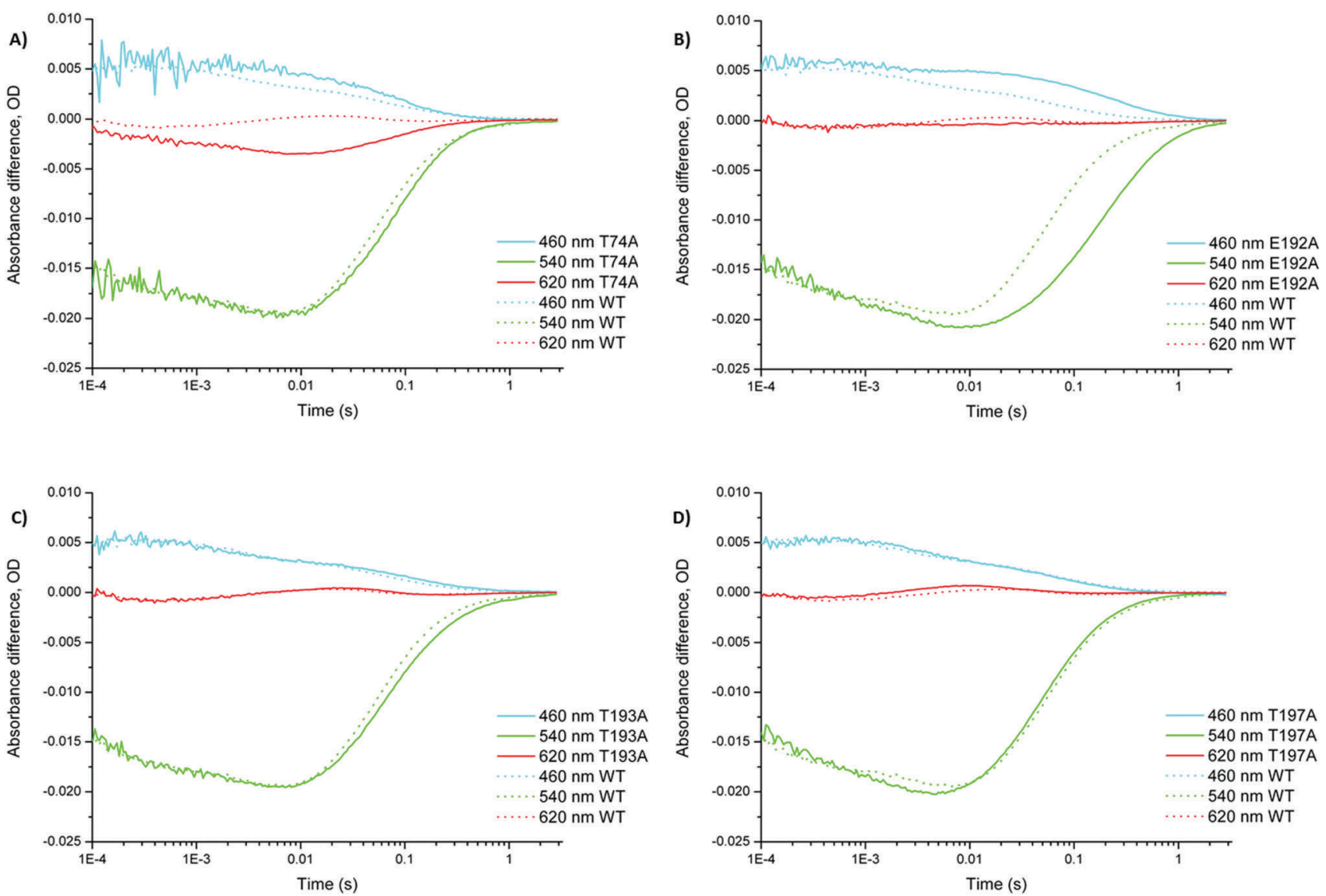

Fig. 11 Comparison of the photocycles of the wild-type MastR (dotted lines) and selected mutants in the extracellular half of the protein (solid lines). The proteins are in $E$. coli membranes encased in polyacrylamide gels, measured at $\mathrm{pH} 6$, buffered by $50 \mathrm{mM} \mathrm{KH}_{2} \mathrm{PO}_{4}, 50 \mathrm{mM} \mathrm{MES}, 0.1 \mathrm{M} \mathrm{NaCl}$, at $22{ }^{\circ} \mathrm{C}$. All traces are normalized based on $540 \mathrm{~nm}$ difference absorption amplitude at $0.7 \mathrm{~ms}$ time point. (A) T74A; (B) E192A; (C) T193A; (D) T197A. 
Asp-85, its putative hydrogen-bonding partner Asn-39 (see the FTIR evidence above), as well as conserved His-166, Ser-211, and Thr-214 (Fig. S1, ESI $\dagger$ ). Ser-211 and Thr-214 are located on the cytoplasmic end of helix G (Fig. 10), with Ser-211 being uniquely conserved in cyanobacterial anion pumps (non-polar in BR and HRs), while Thr-214 is shared with BR (homologous to its Ser-226). One could expect that these residues may form a part of the chloride release pathway, especially considering the known effect of Ser-226 on the cytoplasmic proton conduction in BR. ${ }^{84,103}$ The photocycles of S211A and T214A (Fig. 12) are very similar to each other and differ from the photocycle of the wild-type by a greater apparent accumulation of the $\mathrm{O}$ state and slightly faster photocycle turnover, but are not dramatically different otherwise. This suggests that neither Ser-211 nor Thr-214 are crucially important for chloride transport. The opposite can be stated for His-166, the homolog of Thr-203 of sHR (Thr-218 of pHR), which was strongly implicated (along with Arg-200 in SHR and Lys-215 of pHR) in the chloride release mechanism. ${ }^{28,38,104} \mathrm{Arg}-200$ of SHR is not conserved in cyanobacterial anion pumps, while Thr-203 is present as histidine (His-166 in MastR) and is expected to have an effect on chloride release. Indeed, the photocycle of the H166A MastR mutant is highly perturbed (Fig. 12), showing a very slow decay of the L2 and $\mathrm{N}$ intermediates, which prolongs the photocycle turnover into the seconds time range (by about 1 order of magnitude, $\tau \sim 3.8 \mathrm{~s}$ ). This implies a strong involvement of His-166 in the internal chloride translocation and chloride release to the cytoplasmic bulk, consistent with the expectations from the roles of the homologous threonines in HRs. On the other hand, the role of His-166 could be also a structural one, as it is located one helical turn from the mechanistically important tryptophan in helix F (homolog of Trp-182 of BR), ${ }^{105,106}$ as suggested by modelling (Fig. 10). It is not clear why cyanobacterial anion pumps employ a single histidine in place of the Arg/Lys-Thr pair of HRs, but it points to the uniqueness of this microbial rhodopsin group and confirms its likely evolutionary independence from HRs. Replacement of another conserved residue in the cytoplasmic channel, Asn-39, which is the likely hydrogenbonding partner of Asp-85 (see above), shows an interesting phenotype (Fig. 12). From the earlier (compared to the wildtype) accumulation of the $\mathrm{O}$ state, one could argue that the $\mathrm{L} \rightarrow \mathrm{N} / \mathrm{O}$ transition in the N39A mutant occurs faster, either due to the changed hydrogen-bonding pattern affecting chloride translocation or due to the weaker interhelical hydrogen bonding, which allows for a faster conformational change. On the other hand, the subsequent decay of MastR ${ }^{\prime}$ is delayed, which parallels the
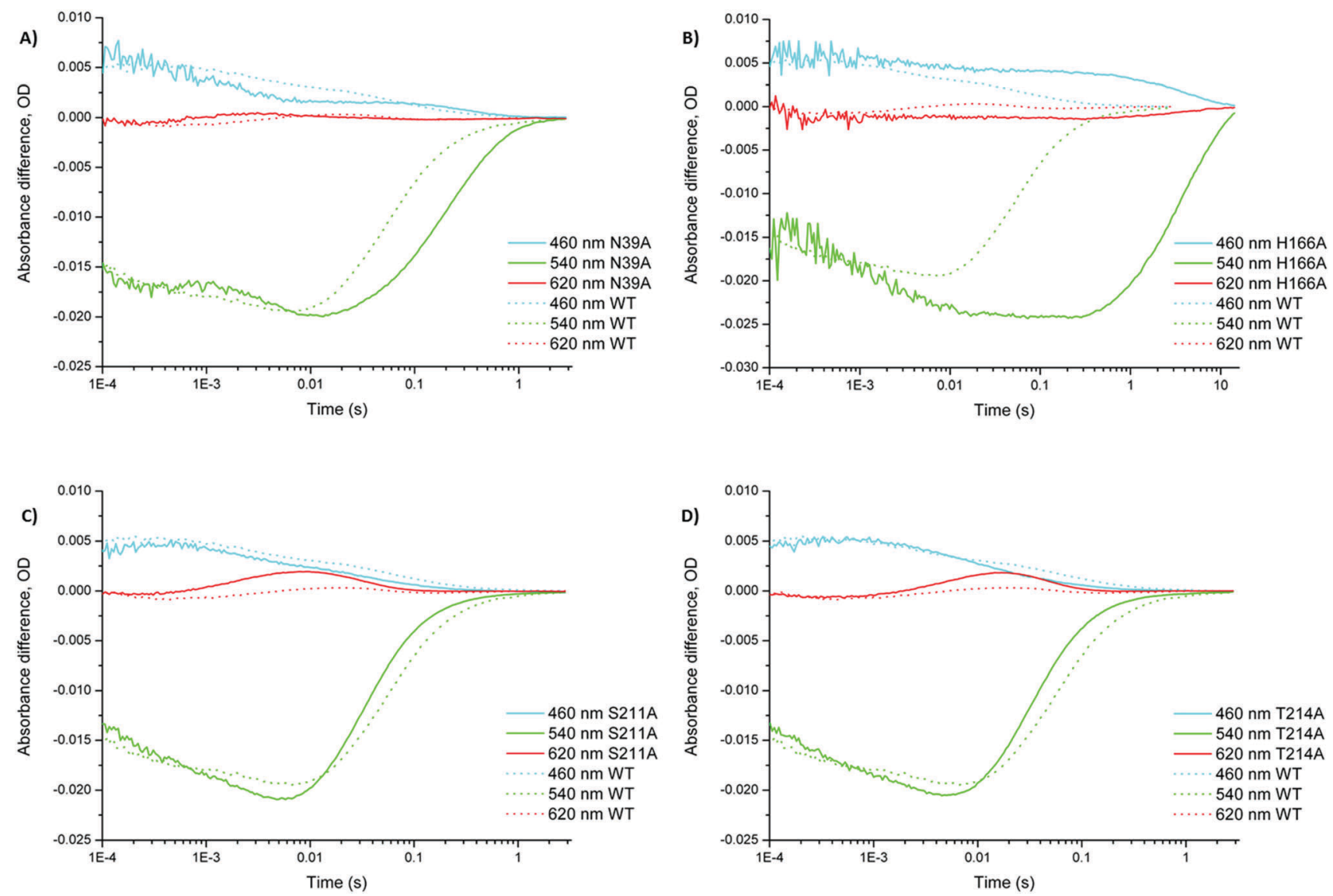

Fig. 12 Comparison of the photocycles of the wild-type MastR (dotted lines) and selected mutants in the cytoplasmic half of the protein (solid lines). The proteins are in $\mathrm{E}$. coli membranes encased in polyacrylamide gels, measured at $\mathrm{pH} 6$, buffered by $50 \mathrm{mM} \mathrm{KH}_{2} \mathrm{PO}_{4}, 50 \mathrm{mM} \mathrm{MES}, 0.1 \mathrm{M} \mathrm{NaCl}$, at $22{ }^{\circ} \mathrm{C}$. All traces are normalized based on $540 \mathrm{~nm}$ difference absorption amplitude at 0.7 ms time point. (A) N39A; (B) H166A, note the different time scale; (C) S211A; (D) T214A. 
situation in the homologous mutant of BR, T46V, in which the $\mathrm{M}$ state decay is accelerated but the $\mathrm{N}$ intermediate decay is dramatically retarded. ${ }^{84,107}$

Finally, the most intriguing question which could be addressed by site-directed mutagenesis is the role of the motif-forming Asp-85, which was shown to transiently deprotonate in the photocycle of MastR (see above, Fig. 6). It is not obvious what to expect, as, on the one hand, according to our FTIR data, Asp-85 showed a robust deprotonation lasting from the L2 intermediate until the end of the photocycle, but on the other hand this residue is not conserved in the cyanobacterial anion pumps group (also present as Val, Ile, Leu) and its replacement does not impair the chloride transport. ${ }^{44}$ Indeed, the photocycle of the D85I mutant of MastR (Fig. 13) is not highly perturbed (isoleucine was chosen to replace aspartate to mimic the replacement observed in the group, Fig. S1, ESI $\dagger$ ). We observed some shift in the N/O equilibrium resulting in a larger accumulation of the $\mathrm{N}$ state and the accompanying modest delay in the photocycle turnover, but the photocycle is not strongly changed otherwise (Fig. 13A). Likewise, the chloride-dependence of the photocycle kinetics of the D85I mutant is very similar to that of the wild-type MastR (Fig. 13B). In view of this, we tried to find conditions under which an effect of the negative charge of transiently deprotonated Asp-85 would become obvious. The presence of negative charge in the homologous position of BR (Asp-96) was shown to stabilize 13-cis states with protonated Schiff base (the $\mathrm{N}$-like states), ${ }^{84,108}$ and the 13-cis conformation of retinal detected in the MastR' state (Fig. 5) suggests that this is the case for MastR as well. Thus, we hypothesized that under conditions where the reprotonation of Asp-85 becomes rate-limiting, the photocycle turnover should be greatly delayed. This is exactly what we observed at pH 9 in the wild-type photocycle (Fig. 13C), which shows large delay of the MastR' decay (more than one order of magnitude) without any effect on the other photocycle steps. Strikingly, this effect is completely eliminated in the D85I mutant (Fig. 13D), which confirms that the reprotonation of Asp-85 is required for the completion of the photocycle. But what could be the possible significance of the transient deprotonation of Asp-85 in the photocycle of MastR? While coordination of chloride and proton movements is known for HRs and chloride-transporting mutants of $\mathrm{BR},{ }^{33,35,39,102}$ the deprotonation of Asp-85 is clearly not required for the chloride transport, as obvious from the D85I mutant behavior (and earlier chloride transport experiments with $\mathrm{D} 85 \mathrm{~N}^{44}$ ). There is an alternative explanation on the utility of
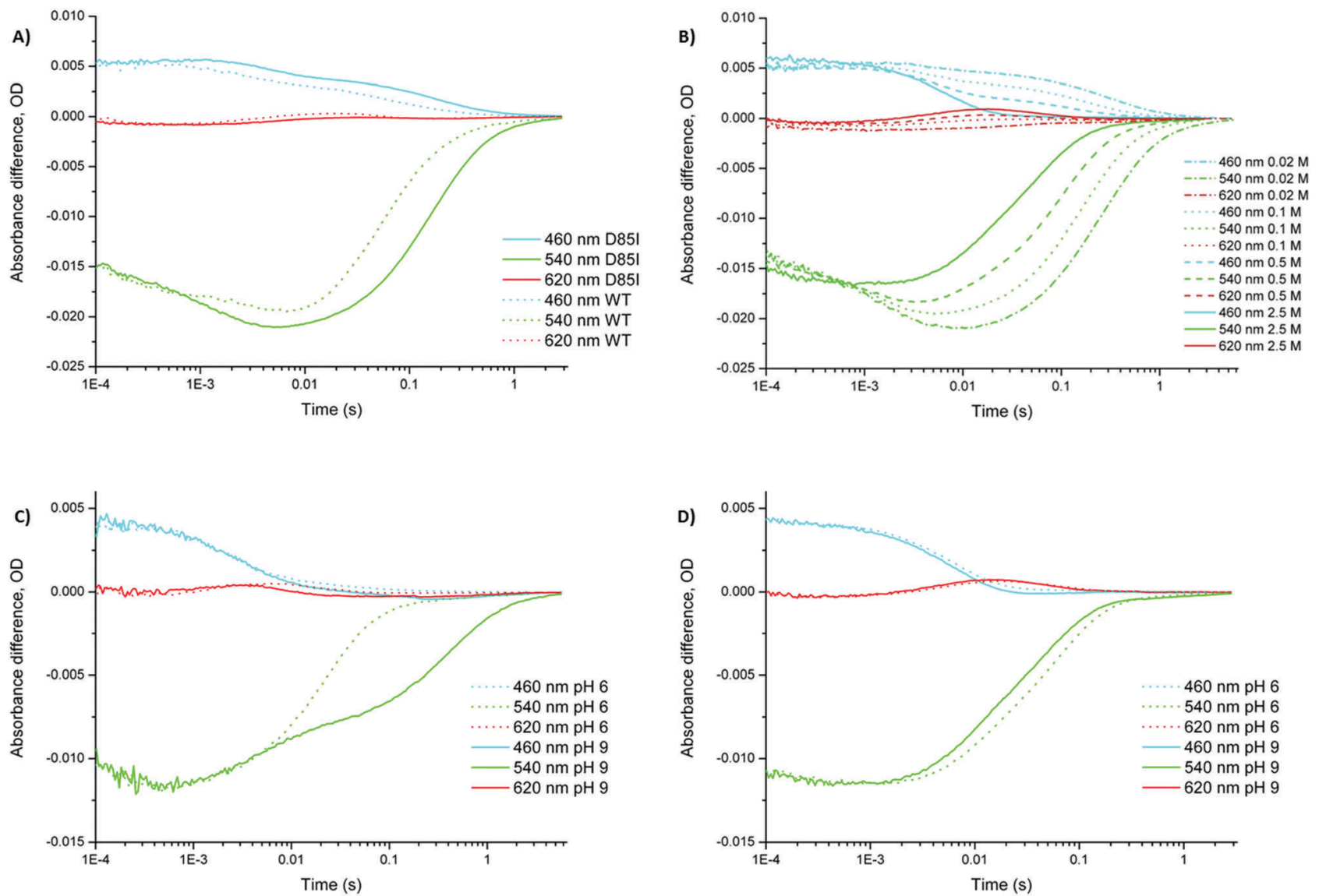

Fig. 13 Comparison of the photocycles of the wild-type MastR and its D85I mutant. The proteins are in E. coli membranes encased in polyacrylamide gels, measured at $22^{\circ} \mathrm{C}$. All traces are normalized based on $540 \mathrm{~nm}$ difference absorption amplitude at 0.7 ms time point. (A) The photocycles of the wild-type (dotted lines) and the D85I mutant (solid lines) at pH 6, buffered by $50 \mathrm{mM} \mathrm{KH}_{2} \mathrm{PO}_{4}, 50 \mathrm{mM} \mathrm{MES}, 0.1 \mathrm{M} \mathrm{NaCl}$; (B) chloride-dependence of the D85I photocycle at $\mathrm{pH} 6, \mathrm{NaCl}$ concentrations as indicated, other conditions as in (A); (C) pH-dependence of the wild-type photocycle, measured in $2.5 \mathrm{M} \mathrm{NaCl}, 50 \mathrm{mM} \mathrm{MES}, 50 \mathrm{mM} \mathrm{KH}_{2} \mathrm{PO}_{4}, 50 \mathrm{mM} \mathrm{CHES}$; (D) pH-dependence of the D85I photocycle, conditions as in (C). 
conserving an aspartate in this position, which seems to be ancestral judging from a large number of the group members preserving it (Fig. S1, ESI $\dagger$ ). We speculate that unlike haloarchaeal HRs, cyanobacterial anion pumps are likely to transport chloride against a very significant electrochemical gradient, and in this process, the anionic aspartate may prevent the backflow of chloride ions in the transiently cytoplasmically open conformation.

\section{Conclusions}

In this paper, we investigated molecular details of the photocycle of a representative of the new group of cyanobacterial anion pumps, MastR. By combining the data from flash-photolysis, vibrational spectroscopy, and site-directed mutagenesis, we came up with the following tentative model of the photocycle, with molecular events in each step summarized below:

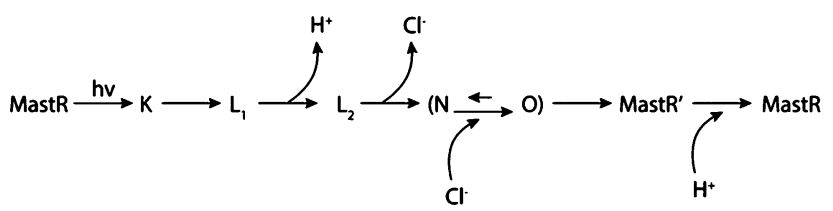

The L1 intermediate likely reflects an initial translocation of chloride ion from the T74/S78/Schiff base binding site towards the cytoplasmic side. We observe the 13-cis conformation of retinal and perturbation of the Schiff base, accompanied by the protein conformational change as can be deduced from the changes in vibrations of cysteines, lipid molecules, and water clusters. The conformational changes propagate further in the L2 state, as reported by changed vibrations of Asn and Arg residues along with further enhancement of Cys bands, causing deprotonation of Asp-85 which creates negative charge in the cytoplasmic channel. We believe that the appearance of this negative charge promotes a chloride ion release to the cytoplasmic bulk in the next step (transition from the L2 state to the N/O mixture), and we see reversal of some of the earlier conformational changes (relaxation of the lipid signals, as well as the signals of Asn, and water clusters) along with perturbation of the backbone amide reflecting this event. Alternatively, chloride ion release could occur in the same step as the proton release, concurrently with Asp-85 deprotonation. The $\mathrm{N}$ to $\mathrm{O}$ transition is probably accompanied by chloride uptake on the extracellular side, as follows from the chloride-dependence of the N/O equilibrium. The final relaxation of protein conformation occurs in the MastR' to MastR transition, in which Asp-85 gets reprotonated, retinal re-isomerizes back to all-trans, and cysteine and arginine perturbations disappear. Importantly, we showed that the reprotonation of Asp-85 is a prerequisite for the photocycle completion. We believe that the transient deprotonation of Asp-85 may be an important regulatory mechanism which makes chloride transport against large electrochemical gradients more favourable energetically.

In summary, our results suggest that the mechanism of anion transport by MastR is unique and involves the interplay of movements of chloride, protons, and water molecules. We believe that cyanobacterial anion pumps are not related to HRs but, more likely, originated from their BR-like ancestors independently. We found a number of molecular features of MastR which support this idea, ranging from the amino acid conservation patterns to the spectroscopic signatures. Central to these is the retention of the ancestral Asp-85, which serves as the cytoplasmic proton donor in BR-like proton pumps, but remains useful as a regulatory element in MastR and many of its close homologs.

\section{Conflicts of interest}

There are no conflicts to declare.

\section{Acknowledgements}

The research was supported by grants from the Canada Foundation for Innovation/Ontario Innovation Trust, the Natural Sciences and Engineering Research Council of Canada (NSERC), and the University of Guelph for L. S. B.; and from the German Research Foundation through SFB-1078, project B3, to J. H. L. S. B. gratefully acknowledges the Faculty Research Visit grant support from DAAD. A. H. is a recipient of Ontario Graduate Scholarship (OGS); A. H.-V. was a recipient of NSERC undergraduate summer research award (USRA). R. M. is a recipient of the $\mathrm{CNPq} / \mathrm{CsF}$ scholarship. A.-N. B. acknowledges financial support from the Excellence Initiative of the German Federal and State governments provided via the Freie Universität Berlin, and from the DFG Collaborative Research Center SFB 1078 Protonation Dynamics in Protein Function, Project C4. L. S. B. wants to thank Dr Yuri I. Wolf (NCBI, NLM, NIH) for fruitful discussions on the evolutionary history of microbial rhodopsins, Dr Víctor A. Lórenz-Fonfría (University of Valencia) for sharing his experience on step-scan FTIR measurements and spectral signatures, and Rachel Hallman for help with flash-photolysis data collection.

\section{Notes and references}

1 W. Humphrey, W. Dalke and K. Schulten, J. Mol. Graphics, 1996, 14, 33-38.

2 F. Sievers, A. Wilm, D. Dineen, T. J. Gibson, K. Karplus, W. Li, R. Lopez, H. McWilliam, M. Remmert, J. Soding, J. D. Thompson and D. G. Higgins, Mol. Syst. Biol., 2011, 7, 539.

3 O. P. Ernst, D. T. Lodowski, M. Elstner, P. Hegemann, L. S. Brown and H. Kandori, Chem. Rev., 2014, 114, 126-163.

4 J. P. Klare, I. Chizhov and M. Engelhard, Results Probl. Cell Differ., 2008, 45, 73-122.

5 E. G. Govorunova, O. A. Sineshchekov, H. Li and J. L. Spudich, Annu. Rev. Biochem., 2017, 86, 845-872.

6 J. K. Lanyi, Annu. Rev. Physiol., 2004, 66, 665-688.

7 U. Haupts, J. Tittor and D. Oesterhelt, Annu. Rev. Biophys. Biomol. Struct., 1999, 28, 367-399.

8 R. A. Mathies, S. W. Lin, J. B. Ames and W. T. Pollard, Annu. Rev. Biophys. Biophys. Chem., 1991, 20, 491-518.

9 J. Herzfeld and J. C. Lansing, Annu. Rev. Biophys. Biomol. Struct., 2002, 31, 73-95. 
10 J. L. Spudich, C. S. Yang, K. H. Jung and E. N. Spudich, Annu. Rev. Cell Dev. Biol., 2000, 16, 365-392.

11 L. S. Brown and K. H. Jung, Photochem. Photobiol. Sci., 2006, 5, 538-546.

12 K. Inoue, Y. Kato and H. Kandori, Trends Microbiol., 2015, 23, 91-98.

13 C. Bamann, E. Bamberg, J. Wachtveitl and C. Glaubitz, Biochim. Biophys. Acta, 2014, 1837, 614-625.

14 J. K. Lanyi and S. P. Balashov, in Halophiles and Hypersaline Environments, ed. A. Ventosa, Springer-Verlag, 2011, ch. 17, pp. 319-340.

15 O. Beja and J. K. Lanyi, Proc. Natl. Acad. Sci. U. S. A., 2014, 111, 6538-6539.

16 S. Yoshizawa, Y. Kumagai, H. Kim, Y. Ogura, T. Hayashi, W. Iwasaki, E. F. DeLong and K. Kogure, Proc. Natl. Acad. Sci. U. S. A., 2014, 111, 6732-6737.

17 L. S. Brown, Biochim. Biophys. Acta, 2014, 1837, 553-561.

18 A. Harris, M. Ljumovic, A. N. Bondar, Y. Shibata, S. Ito, K. Inoue, H. Kandori and L. S. Brown, Biochim. Biophys. Acta, 2015, 1847, 1518-1529.

19 S. P. Balashov, L. E. Petrovskaya, E. S. Imasheva, E. P. Lukashev, A. K. Dioumaev, J. M. Wang, S. V. Sychev, D. A. Dolgikh, A. B. Rubin, M. P. Kirpichnikov and J. K. Lanyi, J. Biol. Chem., 2013, 288, 21254-21265.

20 K. Inoue, M. Konno, R. Abe-Yoshizumi and H. Kandori, Angew. Chem., Int. Ed., 2015, 54, 11536-11539.

21 R. D. Page, Curr. Protoc. Bioinformatics, 2002, ch. 6, unit 6.2.

22 B. Schobert and J. K. Lanyi, J. Biol. Chem., 1982, 257, 10306-10313.

23 K. Ihara, T. Umemura, I. Katagiri, T. Kitajima-Ihara, Y. Sugiyama, Y. Kimura and Y. Mukohata, J. Mol. Biol., 1999, 285, 163-174.

24 A. K. Sharma, D. A. Walsh, E. Bapteste, F. RodriguezValera, W. Ford Doolittle and R. T. Papke, BMC Evol. Biol., 2007, 7, 79.

25 K. Inoue, Y. Nomura and H. Kandori, J. Biol. Chem., 2016, 291, 9883-9893.

26 M. Kolbe, H. Besir, L. O. Essen and D. Oesterhelt, Science, 2000, 288, 1390-1396.

27 T. Kouyama, S. Kanada, Y. Takeguchi, A. Narusawa, M. Murakami and K. Ihara, J. Mol. Biol., 2010, 396, 564-579.

28 M. Rudiger and D. Oesterhelt, EMBO J., 1997, 16, 3813-3821.

29 M. Sato, T. Kikukawa, T. Araiso, H. Okita, K. Shimono, N. Kamo, M. Demura and K. Nitta, J. Biochem., 2003, 134, 151-158.

30 J. Sasaki, L. S. Brown, Y. S. Chon, H. Kandori, A. Maeda, R. Needleman and J. K. Lanyi, Science, 1995, 269, 73-75.

31 J. Tittor, U. Haupts, C. Haupts, D. Oesterhelt, A. Becker and E. Bamberg, J. Mol. Biol., 1997, 271, 405-416.

32 K. Muroda, K. Nakashima, M. Shibata, M. Demura and H. Kandori, Biochemistry, 2012, 51, 4677-4684.

33 G. Varo, L. S. Brown, R. Needleman and J. K. Lanyi, Biochemistry, 1996, 35, 6604-6611.

34 W. Gmelin, K. Zeth, R. Efremov, J. Heberle, J. Tittor and D. Oesterhelt, Photochem. Photobiol., 2007, 83, 369-377.
35 T. Kouyama, H. Kawaguchi, T. Nakanishi, H. Kubo and M. Murakami, Biophys. J., 2015, 108, 2680-2690.

36 G. Varo, R. Needleman and J. K. Lanyi, Biochemistry, 1995, 34, 14500-14507.

37 I. Chizhov and M. Engelhard, Biophys. J., 2001, 81, 1600-1612.

38 M. Sato, M. Kubo, T. Aizawa, N. Kamo, T. Kikukawa, K. Nitta and M. Demura, Biochemistry, 2005, 44, 4775-4784.

39 X. R. Chen, Y. C. Huang, H. P. Yi and C. S. Yang, Biophys. J., 2016, 111, 2600-2607.

40 K. Inoue, F. H. Koua, Y. Kato, R. Abe-Yoshizumi and H. Kandori, J. Phys. Chem. B, 2014, 118, 11190-11199.

41 T. Hosaka, S. Yoshizawa, Y. Nakajima, N. Ohsawa, M. Hato, E. F. DeLong, K. Kogure, S. Yokoyama, T. Kimura-Someya, W. Iwasaki and M. Shirouzu, J. Biol. Chem., 2016, 291, 17488-17495.

42 K. Kim, S. K. Kwon, S. H. Jun, J. S. Cha, H. Kim, W. Lee, J. F. Kim and H. S. Cho, Nat. Commun., 2016, 7, 12677.

43 T. Tsukamoto, S. Yoshizawa, T. Kikukawa, M. Demura and Y. Sudo, J. Phys. Chem. B, 2017, 121, 2027-2038.

44 T. Hasemi, T. Kikukawa, N. Kamo and M. Demura, J. Biol. Chem., 2016, 291, 355-362.

45 A. Niho, S. Yoshizawa, T. Tsukamoto, M. Kurihara, S. Tahara, Y. Nakajima, M. Mizuno, H. Kuramochi, T. Tahara, Y. Mizutani and Y. Sudo, J. Am. Chem. Soc., 2017, 139, 4376-4389.

46 A. Der, P. Hargittai and J. Simon, J. Biochem. Biophys. Methods, 1985, 10, 295-300.

47 S. A. Waschuk, A. G. Bezerra, L. Shi and L. S. Brown, Proc. Natl. Acad. Sci. U. S. A., 2005, 102, 6879-6883.

48 A. K. Dioumaev, Biophys. Chem., 1997, 67, 1-25.

49 T. Resler, B. J. Schultz, V. A. Lorenz-Fonfria, R. Schlesinger and J. Heberle, Biophys. J., 2015, 109, 287-297.

50 L. Shi, I. Kawamura, K. H. Jung, L. S. Brown and V. Ladizhansky, Angew. Chem., Int. Ed., 2011, 50, 1302-1305.

51 V. A. Lorenz-Fonfria and J. Heberle, J. Visualized Exp., 2014, e51622.

52 V. A. Lorenz-Fonfria, T. Resler, N. Krause, M. Nack, M. Gossing, G. Fischer von Mollard, C. Bamann, E. Bamberg, R. Schlesinger and J. Heberle, Proc. Natl. Acad. Sci. U. S. A., 2013, 110, E1273-E1281.

53 B. J. Schultz, H. Mohrmann, V. A. Lorenz-Fonfria and J. Heberle, Spectrochim. Acta, Part A, 2017, 188, 666-674.

54 G. Varo, L. Zimanyi, X. L. Fan, L. Sun, R. Needleman and J. K. Lanyi, Biophys. J., 1995, 68, 2062-2072.

55 Y. Furutani, K. Fujiwara, T. Kimura, T. Kikukawa, M. Demura and H. Kandori, J. Phys. Chem. Lett., 2012, 3, 2964-2969.

56 C. Hackmann, J. Guijarro, I. Chizhov, M. Engelhard, C. Rodig and F. Siebert, Biophys. J., 2001, 81, 394-406.

57 G. Varo, L. S. Brown, J. Sasaki, H. Kandori, A. Maeda, R. Needleman and J. K. Lanyi, Biochemistry, 1995, 34, 14490-14499.

58 K. Ludmann, G. Ibron, J. K. Lanyi and G. Varo, Biophys. J., 2000, 78, 959-966.

59 H. Kakitani, T. Kakitani, H. Rodman, B. Honig and R. Callender, J. Phys. Chem., 1983, 87, 3620-3628.

60 S. O. Smith, J. Lugtenburg and R. A. Mathies, J. Membr. Biol., 1985, 85, 95-109. 
61 T. Baasov, N. Friedman and M. Sheves, Biochemistry, 1987, 26, 3210-3217.

62 S. O. Smith, M. S. Braiman, A. B. Myers, J. A. Pardoen, J. M. L. Courtin, C. Winkel, J. Lugtenburg and R. A. Mathies, J. Am. Chem. Soc., 1987, 109, 3108-3125.

63 S. Gerscher, M. Mylrajan, P. Hildebrandt, M. H. Baron, R. Muller and M. Engelhard, Biochemistry, 1997, 36, 11012-11020.

64 S. P. Fodor, R. A. Bogomolni and R. A. Mathies, Biochemistry, 1987, 26, 6775-6778.

65 Y. S. Chon, J. Sasaki, H. Kandori, L. S. Brown, J. K. Lanyi, R. Needleman and A. Maeda, Biochemistry, 1996, 35, 14244-14250.

66 F. H. M. Koua and H. Kandori, RSC Adv., 2016, 6, 383-392.

67 L. S. Brown, L. Zimanyi, R. Needleman, M. Ottolenghi and J. K. Lanyi, Biochemistry, 1993, 32, 7679-7685.

68 K. Fukuda and T. Kouyama, Biochemistry, 1992, 31, 11740-11747.

69 S. Subramaniam, D. A. Greenhalgh, P. Rath, K. J. Rothschild and H. G. Khorana, Proc. Natl. Acad. Sci. U. S. A., 1991, 88, 6873-6877.

70 K. J. Rothschild, O. Bousche, M. S. Braiman, C. A. Hasselbacher and J. L. Spudich, Biochemistry, 1988, 27, 2420-2424.

71 Y. S. Chon, H. Kandori, J. Sasaki, J. K. Lanyi, R. Needleman and A. Maeda, Biochemistry, 1999, 38, 9449-9455.

72 L. S. Brown, Y. Gat, M. Sheves, Y. Yamazaki, A. Maeda, R. Needleman and J. K. Lanyi, Biochemistry, 1994, 33, 12001-12011.

73 S. O. Smith, J. A. Pardoen, P. P. J. Mulder, B. Curry, J. Lugtenburg and R. Mathies, Biochemistry, 1983, 22, 6141-6148.

74 A. K. Dioumaev, L. S. Brown, J. Shih, E. N. Spudich, J. L. Spudich and J. K. Lanyi, Biochemistry, 2002, 41, 5348-5358.

75 H. Kandori, Y. Yamazaki, M. Hatanaka, R. Needleman, L. S. Brown, H. T. Richter, J. K. Lanyi and A. Maeda, Biochemistry, 1997, 36, 5134-5141.

76 J. M. Pfefferle, A. Maeda, J. Sasaki and T. Yoshizawa, Biochemistry, 1991, 30, 6548-6556.

77 V. Bergo, E. N. Spudich, J. L. Spudich and K. J. Rothschild, Photochem. Photobiol., 2002, 76, 341-349.

78 M. S. Braiman, T. J. Walter and D. M. Briercheck, Biochemistry, 1994, 33, 1629-1635.

79 M. Rudiger, U. Haupts, K. Gerwert and D. Oesterhelt, EMBO J., 1995, 14, 1599-1606.

80 M. Shibata, Y. Saito, M. Demura and H. Kandori, Chem. Phys. Lett., 2006, 432, 545-547.

81 A. Maeda, J. Sasaki, Y. Shichida, T. Yoshizawa, M. Chang, B. F. Ni, R. Needleman and J. K. Lanyi, Biochemistry, 1992, 31, 4684-4690.

82 A. K. Dioumaev, Biochemistry (Moscow), 2001, 66, 1269-1276.

83 A. K. Dioumaev, L. S. Brown, R. Needleman and J. K. Lanyi, Biochemistry, 1999, 38, 10070-10078.
84 A. K. Dioumaev, L. S. Brown, R. Needleman and J. K. Lanyi, Biochemistry, 2001, 40, 11308-11317.

85 M. S. Braiman, O. Bousche and K. J. Rothschild, Proc. Natl. Acad. Sci. U. S. A., 1991, 88, 2388-2392.

86 K. Gerwert and F. Siebert, EMBO J., 1986, 5, 805-811.

87 C. Zscherp and J. Heberle, J. Phys. Chem. B, 1997, 101, 10542-10547.

88 K. Gerwert, B. Hess, J. Soppa and D. Oesterhelt, Proc. Natl. Acad. Sci. U. S. A., 1989, 86, 4943-4947.

89 M. Beck, F. Siebert and T. P. Sakmar, FEBS Lett., 1998, 436, 304-308.

90 L. Shi, S. R. Yoon, A. G. Bezerra, Jr., K. H. Jung and L. S. Brown, J. Mol. Biol., 2006, 358, 686-700.

91 V. A. Lorenz-Fonfria and H. Kandori, J. Am. Chem. Soc., 2009, 131, 5891-5901.

92 S. Wolf, E. Freier, Q. Cui and K. Gerwert, J. Chem. Phys., 2014, 141, 22 D524.

93 J. M. Headrick, E. G. Diken, R. S. Walters, N. I. Hammer, R. A. Christie, J. Cui, E. M. Myshakin, M. A. Duncan, M. A. Johnson and K. D. Jordan, Science, 2005, 308, 1765-1769.

94 J. Breton and E. Nabedryk, Photosynth. Res., 1998, 55, 301-307.

95 C. del Val, L. Bondar and A.-N. Bondar, J. Struct. Biol., 2014, 186, 95-111.

96 A.-N. Bondar, C. del Val, J. A. Freites, D. J. Tobias and S. H. White, Structure, 2010, 18, 847-857.

97 A.-N. Bondar and S. H. White, Biochim. Biophys. Acta, 2012, 1818, 942-950.

98 T. M. Gray and B. W. Matthews, J. Mol. Biol., 1984, 175, 75-81.

99 L. G. Presta and G. D. Rose, Science, 1988, 240, 1632-1641.

100 C. del Val, S. H. White and A.-N. Bondar, J. Membr. Biol., 2012, 245, 717-730.

101 M. Sato, T. Kikukawa, T. Araiso, H. Okita, K. Shimono, N. Kamo, M. Demura and K. Nitta, Biophys. Chem., 2003, 104, 209-216.

102 L. S. Brown, R. Needleman and J. K. Lanyi, Biochemistry, 1996, 35, 16048-16054.

103 T. Marti, H. Otto, T. Mogi, S. J. Rosselet, M. P. Heyn and H. G. Khorana, J. Biol. Chem., 1991, 266, 6919-6927.

104 K. Shibasaki, H. Shigemura, T. Kikukawa, M. Kamiya, T. Aizawa, K. Kawano, N. Kamo and M. Demura, Biochemistry, 2013, 52, 9257-9268.

105 H. Luecke, B. Schobert, H. T. Richter, J. P. Cartailler and J. K. Lanyi, Science, 1999, 286, 255-260.

106 O. Weidlich, B. Schalt, N. Friedman, M. Sheves, J. K. Lanyi, L. S. Brown and F. Siebert, Biochemistry, 1996, 35, 10807-10814.

107 L. S. Brown, Y. Yamazaki, A. Maeda, L. Sun, R. Needleman and J. K. Lanyi, J. Mol. Biol., 1994, 239, 401-414.

108 A. K. Dioumaev, L. S. Brown, R. Needleman and J. K. Lanyi, Biochemistry, 1998, 37, 9889-9893. 